\title{
Reaction rate calculation with time-dependent invariant manifolds
}

\author{
Thomas Bartsch, ${ }^{1}$ F. Revuelta, ${ }^{2}$ R. M. Benito, ${ }^{2}$ and F. Borondo ${ }^{3}$ \\ ${ }^{1}$ Department of Mathematical Sciences, Loughborough University, Loughborough LE11 3TU, United Kingdom \\ ${ }^{2}$ Grupo de Sistemas Complejos and Departamento de Física y Mecánica, Escuela Técnica Superior de \\ Ingenieros Agrónomos, Universidad Politécnica de Madrid, Avda. Complutense s/n, 28040 Madrid, Spain \\ ${ }^{3}$ Departamento de Química, and Instituto Mixto de Ciencias Matemáticas, CSIC-UAM-UC3M-UCM, \\ Universidad Autónoma de Madrid, Cantoblanco, 28049 Madrid, Spain
}

(Received 23 March 2012; accepted 21 May 2012; published online 14 June 2012)

\begin{abstract}
The identification of trajectories that contribute to the reaction rate is the crucial dynamical ingredient in any classical chemical reactivity calculation. This problem often requires a full scale numerical simulation of the dynamics, in particular if the reactive system is exposed to the influence of a heat bath. As an efficient alternative, we propose here to compute invariant surfaces in the phase space of the reactive system that separate reactive from nonreactive trajectories. The location of these invariant manifolds depends both on time and on the realization of the driving force exerted by the bath. These manifolds allow the identification of reactive trajectories simply from their initial conditions, without the need of any further simulation. In this paper, we show how these invariant manifolds can be calculated, and used in a formally exact reaction rate calculation based on perturbation theory for any multidimensional potential coupled to a noisy environment. (C) 2012 American Institute of Physics. [http://dx.doi.org/10.1063/1.4726125]
\end{abstract}

\section{INTRODUCTION}

Transition state theory (TST) provides the conceptual framework for large parts of reaction rate theory. Originally developed to describe the reactivity of small molecules, ${ }^{1-3}$ it was later extended to encompass a wide variety of processes in different branches of science, whose only commonality is a transition from well-defined "reactant" to "product" states. $^{4-13}$ The reason for this success is that TST proposes a simple answer to the two central problems of reaction dynamics: It identifies a reaction mechanism, and provides at the same time a simple approximation to the reaction rate.

More specifically, TST is based on the observation that the rate limiting step in many reactions is the crossing of an energetic barrier. The top of this barrier then forms a bottleneck in the phase space of the reactive system. A reaction can only take place if the barrier is crossed. If a dividing surface (DS) between reactant and product regions of phase space is placed close to the bottleneck, the reaction rate can be computed from the steady-state flux through that surface. A strictly recrossing free DS can be constructed in the phase space of reactive systems with arbitrarily many degrees of freedom. ${ }^{12,14,15}$ The simplest approximation to the rate is then obtained under the assumption that reactive classical trajectories cross the DS only once and never return. This assumption is often appropriate for reactions in the gas phase if the DS is adequately chosen, but even then many reactions strongly violate this assumption. Moreover, if the system is strongly coupled to an environment, for example, a liquid solvent, the no-recrossing assumption is usually impossible to enforce strictly, and often any DS is crossed many times by a typical trajectory. As a result, a TST rate calculation significantly overestimates the reaction rate. For this reason, the focus of TST has long been to construct a DS that eliminates or at least minimizes recrossings (see Ref. 16 for a review).

The recrossing problem can be solved if the reactive trajectories that contribute to the rate can be identified reliably. An obvious means to this end is the numerical simulation of representative trajectories under the influence of the environment. However, such calculations are usually very time consuming. The advantage of the TST approximation is its simplicity. It identifies reactive trajectories simply by noting that they cross the DS from the reactant to the product side. This criterion, which fails if recrossing cannot be ruled out, is easy to use because it only takes account of the instantaneous velocity with which a trajectory crosses the DS. Nevertheless, it raises the prospect of a criterion to identify reactive trajectories simply from their initial conditions, without the need to study their time evolution. In the present paper we will derive such a criterion and demonstrate how it can be used in a rate calculation.

The Langevin equation has been widely used to model the interaction of a reactive system with a surrounding heat bath. ${ }^{17-19}$ Being a classical model, this description neglects quantum effects such as barrier tunnelling, which can be important in the case of light particles, ${ }^{20}$ and the interaction with excited surfaces through conical intersections. ${ }^{21}$ In this setting, Kramers ${ }^{22}$ derived expressions for the rate of escape across a parabolic barrier that apply separately in the limits of weak and strong damping. The generalized Langevin equation is equivalent to a Hamiltonian model in which the reactive system is bilinearly coupled to a bath of harmonic oscillators. ${ }^{23}$ This reformulation allowed extensions of Kramers' rate theory that apply to situations with arbitrary friction ${ }^{24,25}$ or that include corrections due to anharmonic barriers. ${ }^{26-28}$ In this respect, it has long been predicted 
that the rates of activated processes should rise with the coupling to the solvent in the weak coupling regime. However, its direct observation in particle-based models had been elusive because the coupling typically places the processes in the spatial-diffusion limited regime wherein rates decrease with increasing friction. Recently, the Kramers turnover in the rate with microscopic friction has been observed in molecular dynamics trajectories calculation of the $\mathrm{LiNC} \leftrightharpoons \mathrm{LiCN}$ in a bath of Ar atoms. ${ }^{29}$ This observation provided direct and unambiguous evidence for the energy-diffusion regime in which rates increase with friction. In the present work we will not consider any explicit Hamiltonian model for the heat bath; its influence will instead be described by means of a Langevin equation. This approach allows to work within the finitedimensional phase space of the reactive system alone, rather than the infinite-dimensional phase space of the bath. This is advantageous from a computational point of view and also conceptually convenient because the phase space is easier to visualize in low dimension.

The aim of this paper is to describe the geometric phase space structures that allow to classify a trajectory as reactive or nonreactive just by looking at its initial condition, thus avoiding the need of carrying out a numerical simulation. Because the fate of a trajectory with a given initial condition depends on the external force to which it is exposed, any such criterion must take account of the precise realization of that force. A general framework to do that was proposed in a recent series of papers, ${ }^{30-34}$ including the identification of reactive trajectories $^{32}$ and the rate calculation. ${ }^{33}$ It was there shown that the Langevin equation gives rise to a specific trajectory called the transition state (TS) trajectory that remains in the vicinity of the energetic barrier for all times, without ever descending into any of the potential wells. This TS trajectory depends on the realization of the noise, and takes over the role of the fixed saddle point in the conventional TST. A crucial observation in Refs. 30 and 31 for the case of a harmonic barrier is that the dynamics described by the Langevin equation become noiseless when expressed in a time-dependent coordinate system for which the TS trajectory is the moving origin. In the system of relative coordinates it is easy to identify a TST DS that is rigorously free from recrossing. It gives rise to a DS in the original, space fixed coordinate system that is still recrossing-free. This DS is time-dependent since it is attached to the TS trajectory, and it moves through phase space with it. Even more significantly, this construction yields surfaces in phase space that separate reactive from nonreactive trajectories. These surfaces are the stable and unstable manifolds of the TS trajectory, and they also depend on time and on the realization of the noise. Once they are known, initial conditions on one side of the surface are immediately classified as reactive, while those on the other side are nonreactive. Thus, the existence of these invariant manifolds solves the diagnostic problem of standard rate theory that was explained above. They were used in Ref. 33 to obtain a compact rate formula, strictly valid only for harmonic barriers. An ad hoc application to systems with an anharmonic barrier produced, however, promising results. ${ }^{32,33}$

In the present paper, we develop a rigorous generalization of the time-dependent TST formalism applicable to anharmonic barriers using perturbation theory. We show that the invariant manifolds persist in anharmonic systems and, more importantly, they retain the ability to distinguish between reactive and nonreactive trajectories, thus determining the chemical reactivity of the system. Finally, a simple perturbative scheme that allows one to calculate the invariant manifolds for a specific anharmonic potential barrier will be presented, and it will be used to obtain an analytic expansion for the reaction rate. In the first part of the paper, we restrict our study to the one-dimensional case. In this situation, the finite barrier corrections that were obtained in Refs. 26-28 will be recovered. We have already given a brief account of these results in Ref. 35. We will here supply the details of the calculation that could not be presented within the confines of a Communication. We will then introduce the modifications to the theory that are necessary to accommodate multidimensional reactive systems. The efficacy of our method is demonstrated by deriving the first- and second-order corrections to the reaction rate in the two-dimensional model potential already used in Refs. 32 and 33.

A final point is worth commenting on in this Introduction. Perturbative rate calculations on multidimensional anharmonic barriers have also been recently reported in Refs. 36-39. As in the present work, these authors based their work on the identification of the TS trajectory for the harmonic limit in Refs. 30 and 31. Our work, however, goes beyond those previous results in two main respects. First, and most importantly, it provides an explicit and detailed description of the invariant geometric structures in phase space that govern the reaction dynamics, rather than studying them implicitly through approximate invariants and their imprint on an ensemble of trajectories. Second, whereas the normal form procedure in Refs. 36-39 aims at constructing a coordinate system in which the dynamics in the neighborhood of the barrier can be simplified in general terms, we derive a version of the perturbation theory that is specifically directed at calculating the invariant manifolds that are relevant to reaction rate theory. This perturbative scheme can therefore be much simpler, and permits the analytical computation of corrections to Kramers' transmission factor for anharmonic potentials. Indeed, the calculation of the invariant manifolds can be easily carried out by hand, whereas a normal form transformation always requires computer assistance. This ease of computation makes the invariant manifolds an attractive tool for practical rate calculations.

The outline of the paper is as follows. In Sec. II we present the basic definitions and results of rate theory that will be used to develop our method. Section III is devoted to a qualitative description of the invariant manifolds that give structure to the dynamics in the vicinity of an energy barrier, and Sec. IV presents a method for their calculation. In Sec. $\mathrm{V}$ a general expression for the reaction rate in the case of an anharmonic barrier is derived. A description of the statistical properties of the invariant manifolds that are required to evaluate the rate formula and the perturbative and numerical results for various one-dimensional potentials are also given. Finally, in Sec. VI we discuss the modifications to the foregoing developments that are required in multidimensional systems, and we also present 
results for the reaction rate on an anharmonic twodimensional barrier.

\section{FUNDAMENTALS OF RATE THEORY}

In this section we outline the fundamentals of reaction rate theory that will be used in the rest of the paper. The reader is referred to Refs. 17-19 for more details.

We assume that the reactant and product regions in configuration space are separated by a DS that is characterized by the value $x=x^{\ddagger}$ of a generalized reaction coordinate $x$, which we choose such that the product region is given by $x>$ $x^{\ddagger}$. The reaction rate is then given by the flux-over-population expression

$$
k=\frac{J}{N},
$$

where $N$ is the average population of the reactant region and

$$
J=\left\langle v_{x} \chi_{\mathrm{r}}\left(v_{x}, \boldsymbol{q}_{\perp}, \boldsymbol{v}_{\perp}\right)\right\rangle_{\alpha, \mathrm{IC}}
$$

is the reactive flux out of that region. Here, $v_{x}$ denotes the velocity component perpendicular to the DS, $\boldsymbol{q}_{\perp}$ is the coordinates within the surface, and $\boldsymbol{v}_{\perp}$ is the corresponding velocities. The characteristic function $\chi_{\mathrm{r}}\left(v_{x}, \boldsymbol{q}_{\perp}, \boldsymbol{v}_{\perp}\right)$ takes the value 1 if the trajectory starting at $x=x^{\ddagger}, v_{x}, \boldsymbol{q}_{\perp}, \boldsymbol{v}_{\perp}$ is reactive, i.e., moves to products for large times, and 0 otherwise. Its purpose is to ensure that only reactive trajectories contribute to the reactive flux. The average in Eq. (2) extends over the realizations, $\alpha$, of the external noise and over a thermal equilibrium ensemble of initial conditions that are constrained to lie on the DS. The latter ensemble is described by a probability density function

$$
p\left(x, v_{x}, \boldsymbol{q}_{\perp}, \boldsymbol{v}_{\perp}\right)=\delta\left(x-x^{\ddagger}\right) \exp \left(-\frac{v_{x}^{2}}{2 k_{\mathrm{B}} T}\right) p_{\perp}\left(\boldsymbol{q}_{\perp}, \boldsymbol{v}_{\perp}\right),
$$

which includes a Boltzmann distribution of the velocities $v_{x}$ and a Boltzmann distribution

$$
p_{\perp}\left(\boldsymbol{q}_{\perp}, \boldsymbol{v}_{\perp}\right)=\frac{1}{Z} \exp \left(-\frac{\boldsymbol{v}_{\perp}^{2} / 2+U\left(x^{\ddagger}, \boldsymbol{q}_{\perp}\right)}{k_{\mathrm{B}} T}\right)
$$

of the transverse coordinates and velocities. The factor $Z$ in Eq. (4) is the partition function of the transverse motion. It ensures that

$$
\int d \boldsymbol{q}_{\perp} d \boldsymbol{v}_{\perp} p\left(\boldsymbol{q}_{\perp}, \boldsymbol{v}_{\perp}\right)=1 .
$$

In Eq. (3) we have used mass-scaled coordinates and we have left out an overall normalization factor. In particular, we did not include the Arrhenius factor

$$
\exp \left(-\frac{\Delta E^{\ddagger}}{k_{\mathrm{B}} T}\right)
$$

that includes the activation energy $\Delta E^{\ddagger}$ of the reaction. The overall normalization of the distribution function is well understood, and it is irrelevant to the calculation of the transmission factor (7) below, on which we will focus in this work. For simplicity, we can therefore work with the unnormalized distribution function (3).
The characteristic function $\chi_{\mathrm{r}}$ in Eq. (2) encodes the entire complexity of the reaction dynamics on an anharmonic barrier. The main task of a reaction rate calculation is to evaluate this function. In general, this can only be achieved by a numerical simulation. A simple approximation to this crucial ingredient is provided by TST. It assumes that no trajectory can cross the DS more than once. As a consequence, every trajectory that crosses the DS from the reactant to the product side must be reactive, every trajectory that crosses in the opposite direction must be nonreactive. To implement this approximation, we replace the characteristic function in Eq. (2) by

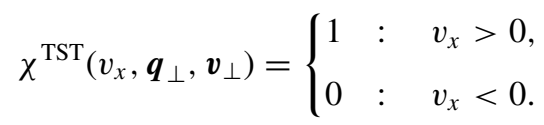

This gives rise to the TST approximation to the rate constant

$$
k^{\mathrm{TST}}=\frac{\left\langle v_{x} \chi^{\mathrm{TST}}\left(v_{x}, \boldsymbol{q}_{\perp}, \boldsymbol{v}_{\perp}\right)\right\rangle_{\mathrm{IC}}}{N},
$$

in which the average over the noise $\alpha$ can be suppressed because $\chi^{\text {TST }}$ does not depend on it.

When the no-recrossing assumption of TST is not satisfied, the approximation (6) will overestimate the rate, often by a large factor. To quantify the effects of non-TST behavior, a transmission factor,

$$
\kappa=\frac{k}{k^{\mathrm{TST}}} \leq 1,
$$

is introduced that relates the exact rate to the TST approximation. It can be obtained from the ratio of the flux across the barrier to its TST approximation:

$$
\kappa=\frac{\left\langle v_{x} \chi_{\mathrm{r}}\left(v_{x}, \boldsymbol{q}_{\perp}, \boldsymbol{v}_{\perp}\right)\right\rangle_{\alpha, \mathrm{IC}}}{\left\langle v_{x} \chi^{\mathrm{TST}}\left(v_{x}, \boldsymbol{q}_{\perp}, \boldsymbol{v}_{\perp}\right)\right\rangle_{\mathrm{IC}}} .
$$

To evaluate (7) numerically, one can randomly sample initial conditions and noise sequences from the appropriate ensembles, and simulate the behavior of each trajectory until its energy is so far below the barrier top that it can be regarded as having been thermalized on either the reactant or the product side of the barrier. The trajectory can then be classified as reactive or non-reactive depending on what state it reached. All numerical results presented in this work were obtained in this way.

This algorithm is conceptually straightforward, but computationally costly. It would be highly desirable to find a criterion that allows one to identify the reactive trajectories without having to carry out a numerical simulation. Sections III-VI will describe the phase space structures that will provide such a criterion.

\section{TIME-DEPENDENT INVARIANT MANIFOLDS}

\section{A. The Langevin model}

We begin by specifying the model that will be used. The Langevin equation describes the reduced dynamics of a lowdimensional system coupled to an external heat bath. ${ }^{17}$ It is given by

$$
\ddot{\boldsymbol{q}}=-\nabla_{\boldsymbol{q}} U(\boldsymbol{q})-\boldsymbol{\Gamma} \dot{\boldsymbol{q}}+\boldsymbol{\xi}_{\alpha}(t),
$$


where $\boldsymbol{q}$ is an $N$ dimensional vector of mass-scaled coordinates, $U(\boldsymbol{q})$ is the potential of mean force, $\boldsymbol{\Gamma}$ is a symmetric positive-definite $N \times N$ matrix of damping constants, and $\xi_{\alpha}(t)$ is the fluctuating force exerted by the heat bath. It is connected to the friction matrix $\boldsymbol{\Gamma}$ by the fluctuation-dissipation theorem ${ }^{40}$

$$
\left\langle\xi_{\alpha}(t) \xi_{\alpha}^{\mathrm{T}}\left(t^{\prime}\right)\right\rangle_{\alpha}=2 k_{\mathrm{B}} T \Gamma \delta\left(t-t^{\prime}\right),
$$

where $k_{\mathrm{B}}$ is the Boltzmann constant and $T$ is the temperature. Throughout most of this work, we consider a one-dimensional problem in which the friction matrix $\boldsymbol{\Gamma}$ simply reduces to a scalar $\gamma$, and the position vector $\boldsymbol{q}$ contains a single coordinate $x$. If we expand the potential of mean force around its saddle point, we can write it as

$$
U(x)=-\frac{1}{2} \omega_{\mathrm{b}}^{2} x^{2}+\varepsilon \frac{c_{3}}{3} x^{3}+\varepsilon^{2} \frac{c_{4}}{4} x^{4}+\cdots,
$$

where $\varepsilon$ is a formal perturbation parameter that serves only to keep track of the orders of perturbation theory, and finally will be set to $\varepsilon=1$. For the mean force itself we write

$$
-\frac{\mathrm{d} U}{\mathrm{~d} x}=\omega_{\mathrm{b}}^{2} x+f(x),
$$

where $f(x)$ denotes the anharmonic parts of the force.

\section{B. Time-dependent transition states}

Because the Langevin equation (8) is a second-order differential equation, its phase space is two-dimensional, with coordinates $x$ and $v_{x}=\dot{x}$. As it was observed in Refs. 30-32, the dynamics of the Langevin equation in the harmonic approximation can be diagonalized by rewriting it in coordinates $u$ and $s$ given by

$$
u=\frac{v_{x}-\lambda_{\mathrm{s}} x}{\lambda_{\mathrm{u}}-\lambda_{\mathrm{s}}}, \quad s=\frac{-v_{x}+\lambda_{\mathrm{u}} x}{\lambda_{\mathrm{u}}-\lambda_{\mathrm{s}}},
$$

or

$$
x=u+s, \quad v_{x}=\lambda_{\mathrm{u}} u+\lambda_{\mathrm{s}} s .
$$

The constants

$$
\lambda_{\mathrm{s}, \mathrm{u}}=-\frac{1}{2}\left(\gamma \pm \sqrt{\gamma^{2}+4 \omega_{\mathrm{b}}^{2}}\right)
$$

are the eigenvalues that arise in the diagonalization. They satisfy $\lambda_{\mathrm{s}}<0<\lambda_{\mathrm{u}}$ and

$$
\lambda_{\mathrm{u}}+\lambda_{\mathrm{s}}=-\gamma, \quad \lambda_{\mathrm{u}} \lambda_{\mathrm{s}}=-\omega_{\mathrm{b}}^{2} .
$$

In the new set of coordinates, the equations of motion read

$$
\begin{aligned}
& \dot{u}=\lambda_{\mathrm{u}} u+\frac{f(x)}{\lambda_{\mathrm{u}}-\lambda_{\mathrm{s}}}+\frac{1}{\lambda_{\mathrm{u}}-\lambda_{\mathrm{s}}} \xi_{\alpha}(t), \\
& \dot{s}=\lambda_{\mathrm{s}} s-\frac{f(x)}{\lambda_{\mathrm{u}}-\lambda_{\mathrm{s}}}-\frac{1}{\lambda_{\mathrm{u}}-\lambda_{\mathrm{s}}} \xi_{\alpha}(t) .
\end{aligned}
$$

These equations decouple in the harmonic approximation, i.e., if $f(x)=0$, but they are still subject to the time-dependent stochastic driving force $\xi_{\alpha}(t)$. This time dependence can be removed by the coordinate shift

$$
\Delta u=u-u^{\ddagger}, \quad \Delta s=s-s^{\ddagger},
$$

where

$u^{\ddagger}(t)=\frac{1}{\lambda_{\mathrm{u}}-\lambda_{\mathrm{s}}} S\left[\lambda_{\mathrm{u}}, \xi_{\alpha} ; t\right], \quad S^{\ddagger}(t)=-\frac{1}{\lambda_{\mathrm{u}}-\lambda_{\mathrm{s}}} S\left[\lambda_{\mathrm{s}}, \xi_{\alpha} ; t\right]$,

and the $S$ functionals ${ }^{30,41}$ are given by

$$
S_{\tau}[\mu, g ; t]= \begin{cases}-\int_{t}^{\infty} g(\tau) \exp (\mu(t-\tau)) d \tau & : \operatorname{Re} \mu>0, \\ +\int_{-\infty}^{t} g(\tau) \exp (\mu(t-\tau)) d \tau & : \operatorname{Re} \mu<0 .\end{cases}
$$

The subscript $\tau$ is used in the $S$ functional to indicate the integration variable. This subscript will be left out whenever this does not cause any ambiguities. Similarly, we have for the sake of simplicity not indicated in our notation that $u^{\ddagger}(t)$ and $s^{\ddagger}(t)$ depend on the realization $\alpha$ of the noise, although they both obviously do.

The functions $u^{\ddagger}(t)$ and $s^{\ddagger}(t)$ solve the equations of motion in the harmonic limit $f(x)=0$. They can therefore be regarded as the coordinates of a special trajectory called the TS trajectory. This trajectory is distinguished from all other trajectories that are exposed to the same noise by the fact that it remains in the vicinity of the saddle point for all times, whereas a typical trajectory would descend into either the reactant or the product well both in the remote past and in the distant future. Accordingly, when using coordinates $\Delta u$ and $\Delta s$, we are describing a trajectory relative to the TS trajectory, which acts as a moving coordinate origin. In what follows, we will refer to $\Delta u$ and $\Delta s$ as relative coordinates and to the original $u$ and $s$, or $x$ and $v_{x}$ as space fixed coordinates.

The equations of motion in relative coordinates are

$$
\begin{gathered}
\Delta \dot{u}=\lambda_{\mathrm{u}} \Delta u+\frac{f(x)}{\lambda_{\mathrm{u}}-\lambda_{\mathrm{s}}}, \\
\Delta \dot{s}=\lambda_{\mathrm{s}} \Delta s-\frac{f(x)}{\lambda_{\mathrm{u}}-\lambda_{\mathrm{s}}} .
\end{gathered}
$$

At first sight, it appears that the time-dependent and stochastic shift (16) has removed both the time-dependence and the dependence on the realization $\alpha$ of the noise. However, this is only true in the harmonic approximation. If we express the position coordinate $x$ in terms of the relative coordinates $\Delta u$ and $\Delta s$ as

$$
x=x^{\ddagger}+\Delta u+\Delta s,
$$

with $x^{\ddagger}=u^{\ddagger}+s^{\ddagger}$, Eq. (19) turns into

$$
\begin{aligned}
& \Delta \dot{u}=\lambda_{\mathrm{u}} \Delta u+\frac{f\left(x^{\ddagger}+\Delta u+\Delta s\right)}{\lambda_{\mathrm{u}}-\lambda_{\mathrm{s}}}, \\
& \Delta \dot{s}=\lambda_{\mathrm{s}} \Delta s-\frac{f\left(x^{\ddagger}+\Delta u+\Delta s\right)}{\lambda_{\mathrm{u}}-\lambda_{\mathrm{s}}} .
\end{aligned}
$$

The position $x^{\ddagger}(t)$ of the TS trajectory represents a timedependent stochastic driving in these equations of motion. Nevertheless, the coordinate shift has removed the stochastic driving from the leading-order terms in Eq. (21) and pushed it into the anharmonic perturbation. 
The description of the geometric phase space structure in the vicinity of the saddle point is most easily done if one starts from the harmonic limit. A full discussion can be found in Refs. 30 and 31. The equations of motion (19) decouple and become time independent when $f(x)=0$, and they can then be easily solved by writing

$$
\begin{gathered}
\Delta u(t)=\Delta u(0) e^{\lambda_{\mathrm{u}} t}, \\
\Delta s(t)=\Delta s(0) e^{\lambda_{\mathrm{s}} t} .
\end{gathered}
$$

Since $\lambda_{\mathrm{u}}>0$ and $\lambda_{\mathrm{s}}<0$, the coordinate $\Delta u$ grows exponentially in time, whereas $\Delta s$ shrinks. Therefore, $\Delta u$ and $\Delta s$ correspond to unstable and stable directions in phase space, respectively. In particular, the lines $\Delta u=0$ and $\Delta s=0$ are invariant under the dynamics. A trajectory that starts on the line $\Delta u=0$ will asymptotically approach the origin as $t \rightarrow \infty$; this line is called the stable manifold of the origin. A trajectory on the line $\Delta s=0$ will move away from the origin as $t \rightarrow \infty$, but it will approach the origin as $t \rightarrow-\infty$; this line is called the unstable manifold of the origin.

The stable and unstable manifolds of the origin, together with several typical trajectories in relative coordinates, are shown in Fig. 1(a). The invariant manifolds separate trajectories with different qualitative behavior. Trajectories above the stable manifold, i.e., with larger relative velocity, move to the product side of the barrier for asymptotically long times, whereas trajectories below the stable manifold move to the reactant side. Similarly, trajectories above the unstable manifold come from the reactant side in the distant past, whereas
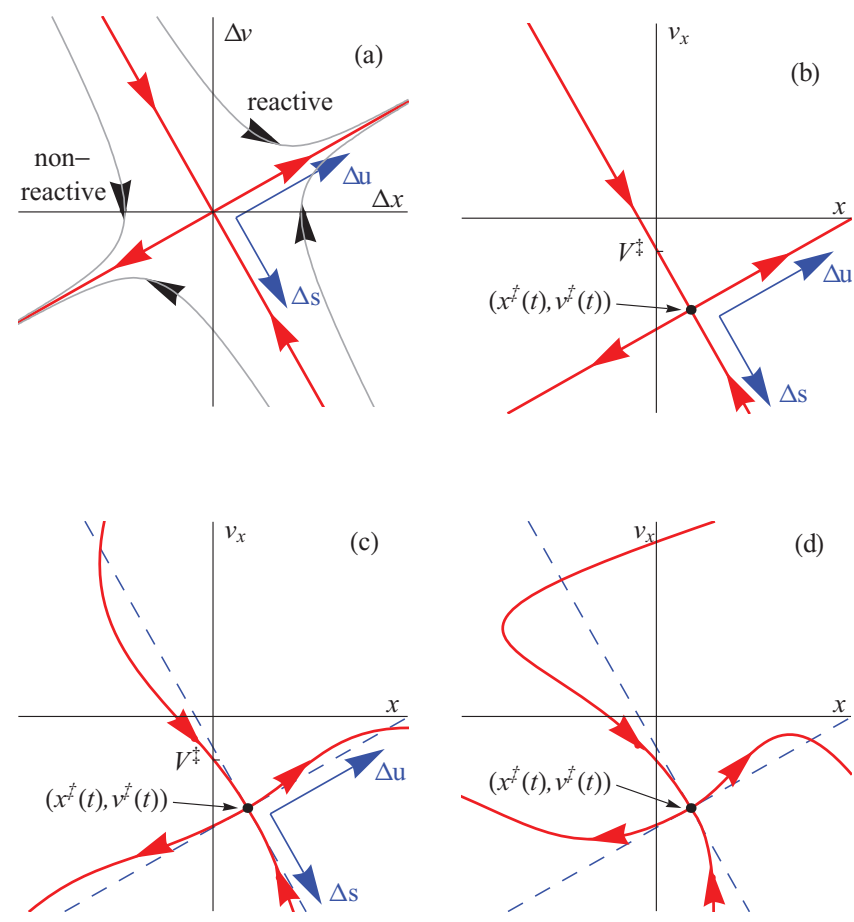

FIG. 1. Phase space view of the time-dependent invariant manifolds of the Langevin equation. (a) Invariant manifolds are time-independent in the harmonic approximation and in relative coordinates. (b) In space-fixed coordinates, the invariant manifolds are attached to the TS trajectory and move through phase space with it. (c) Anharmonic coupling deforms the manifolds. Both their position and their shape are stochastically time dependent. (d) Invariant manifolds can deviate strongly from the harmonic approximation if the anharmonicities are strong. trajectories below the unstable manifold come from the product side.

For a reaction rate calculation we need to ascertain whether a trajectory will turn into reactants or products in the future. In our approach this sentence is rephrased into the condition: We need to decide whether a trajectory lies above or below the stable manifold. In other words, the stable manifold encodes the information about the reaction dynamics that is most relevant to us. We will therefore focus on the stable manifold in what follows, largely ignoring the unstable manifold.

We can return to space fixed coordinates by undoing the time-dependent shift (16). After the shift, the stable and unstable manifolds are not attached to the origin of the coordinate system any more, but instead to the TS trajectory as a moving origin, as shown in Fig. 1(b). Since the TS trajectory is time dependent, the manifolds will move through phase space with it. Nevertheless, they still separate trajectories with different asymptotic behaviors. Given a trajectory with a given initial condition at a certain time, it can be classified as reactive or non-reactive by knowing the instantaneous position of the stable manifold at that time. Through the TS trajectory, that instantaneous position will depend on the realization of the noise.

It is clear from Fig. 1(b) that at any time and for any realization of the noise the stable manifold intersects the axis $x=0$ at a point with a velocity $V^{\ddagger}$. Trajectories with initial positions $x=0$ and initial velocities $v_{x}>V^{\ddagger}$ are reactive, while trajectories with initial velocities $v_{x}<V^{\ddagger}$ are not. The critical velocity $V^{\ddagger}$ depends on time and on the realization of the noise. For the harmonic approximation, it was shown in Ref. 33, and it will be rederived below, that

$$
V^{\ddagger} \equiv V_{0}^{\ddagger}=\left(\lambda_{\mathrm{u}}-\lambda_{\mathrm{s}}\right) u^{\ddagger}(0) .
$$

Since the critical velocity characterizes reactive trajectories, the transmission factor (7) can be expressed in terms of $V^{\ddagger}$ (see Ref. 33 and Sec. V below).

This picture of the invariant manifolds was introduced in Refs. 30 and 31 and applied to rate calculations in Refs. 32 and 33. The main purpose of the present work is to explore how this picture changes when anharmonicities of the barrier potential are taken into account. In this case the equations of motion (19) are coupled in a nonlinear time-dependent way, and they cannot be solved easily. However, as long as the coupling is sufficiently weak, one can expect to find a TS trajectory with its associated stable and unstable manifolds that are close to those in the harmonic approximation. Indeed, there are general theorems in the theory of stochastic dynamical systems ${ }^{42}$ that guarantee the persistence of these structures. As shown in Fig. 1(c), the invariant manifolds in an anharmonic system will be tangent to their harmonic approximations at the TS trajectory, but they will not be straight lines anymore. Because the coupling term in Eqs. (19) is stochastically time dependent, the shapes of the invariant manifolds as well as their positions in phase space depend on time and on the realization of the noise.

The intersection of the stable manifold with the axis $x=0$ will give rise to a critical velocity $V^{\ddagger}$ such that trajectories with initial velocities larger than $V^{\ddagger}$ will be reactive, 
those with smaller initial velocities will not. The critical velocity can therefore be used in a rate calculation in an anharmonic system just as it can in the harmonic approximation, though its value will be different from (23). A method to calculate the critical velocity will be developed in Sec. IV.

In general it cannot be guaranteed that there will only be a single intersection between the stable manifold and the axis $x=0$. In fact, if the reaction potential has wells on the reactant and/or product side of the barrier, it is likely that there will be further intersections, as illustrated in Fig. 1(d). If a trajectory on the stable manifold is followed backwards in time, it will descend from the barrier, settling in one of the wells for some time. If it is followed for long enough, it will eventually cross the barrier again into the other well. In doing so, it must cross the line $x=0$ again, and thus give rise to additional intersections between the stable manifold and that line. However, as these additional intersections stem from previous barrier crossings, they must be neglected in the rate calculation. Only for extremely strong nonlinearities additional intersections that are not separated by periods in which the trajectory was equilibrated in one of the wells will be found. We will neglect that possibility in what follows. Instead, we will apply perturbation theory to calculate a value for the critical velocity that reduces to its harmonic approximation in the appropriate limit.

The TS trajectory (17) solves the equations of motion (15) in the harmonic limit, but not in the presence of anharmonic coupling. Strictly speaking, therefore, Eq. (17) does not define a TS trajectory on an anharmonic potential. Such a trajectory could be obtained by a perturbative expansion similar to the one to be developed in Sec. IV. For our purposes, however, this will not be necessary. The harmonic TS trajectory forms a suitable basis for the perturbation theory. We will therefore use the notation $u^{\ddagger}, s^{\ddagger}$, and $x^{\ddagger}$ exclusively to denote the harmonic approximation to the TS trajectory.

\section{PERTURBATIVE CALCULATION OF THE STABLE MANIFOLD}

The critical velocity is defined by the intersection of the line $x=0$ with the stable manifold of the TS trajectory. The stable manifold contains all those trajectories that approach the TS trajectory as $t \rightarrow \infty$. They remain bounded for large times. Solutions to the equations of motion (21) that satisfy this boundary condition at large time lie on the stable manifold.

Equation (21a) can be formally solved in terms of the $S$ functional (18) as

$$
\Delta u(t)=C e^{\lambda_{\mathrm{u}} t}+\frac{1}{\lambda_{\mathrm{u}}-\lambda_{\mathrm{s}}} S\left[\lambda_{\mathrm{u}}, f\left(x^{\ddagger}+\Delta u+\Delta s\right) ; t\right] .
$$

Notice that this is only a formal solution due to the presence of the unknown function $\Delta u$ in the r.h.s. of the equation. Furthermore, the $S$ functional is undefined for most trajectories, only existing for the trajectories that remain bounded in the remote future. However, these are precisely the trajectories we are interested in. For consistency, we must then set $C=0$, just as was done in Refs. 30 and 31 in the construction of the TS trajectory. A trajectory on the unstable manifold therefore satisfies the integral equation

$$
\Delta u(t)=\frac{1}{\lambda_{\mathrm{u}}-\lambda_{\mathrm{s}}} S\left[\lambda_{\mathrm{u}}, f\left(x^{\ddagger}+\Delta u+\Delta s\right) ; t\right] .
$$

This expression automatically incorporates the boundary condition at $t \rightarrow \infty$ that we wish to impose.

For the stable component, we might be tempted to use the analogous formal solution

$$
\Delta s(t)=C e^{\lambda_{\mathrm{s}} t}-\frac{1}{\lambda_{\mathrm{u}}-\lambda_{\mathrm{s}}} S\left[\lambda_{\mathrm{s}}, f\left(x^{\ddagger}+\Delta u+\Delta s\right) ; t\right] .
$$

However, the $S$ functional for a negative eigenvalue depends on the infinite past of its argument and is well defined only for trajectories that remain bounded in the past. Most trajectories on the stable manifold, except for the TS trajectory itself, will not satisfy this condition. This difficulty can be circumvented by using the modified $S$ functional

$$
\bar{S}_{\tau}[\mu, g ; t]=\int_{0}^{t} g(\tau) e^{\mu(t-\tau)} d \tau
$$

that is well defined for all values of $\mu$. It satisfies the differential equation

$$
\frac{d}{d t} \bar{S}[\mu, g ; t]=\mu \bar{S}[\mu, g ; t]+g(t)
$$

and the initial condition $\bar{S}[\mu, g ; 0]=0$. With this functional, a formal solution to the equation of motion (21b) can be written as

$$
\Delta s(t)=\Delta s(0) e^{\lambda_{\mathrm{s}} t}-\frac{1}{\lambda_{\mathrm{u}}-\lambda_{\mathrm{s}}} \bar{S}\left[\lambda_{\mathrm{s}}, f\left(x^{\ddagger}+\Delta u+\Delta s\right) ; t\right] .
$$

Note that this integral equation does not impose any boundary condition on the function $\Delta s$, thus leaving free choice of the initial condition $\Delta s(0)$.

The critical velocity $V^{\ddagger}$ is determined by the condition that the trajectory with initial conditions $x(0)=0$ and $v(0)$ $=V^{\ddagger}$ satisfies the integral equations (24) and (26). The first one of these conditions can be rewritten as

$$
\Delta s(0)=-x^{\ddagger}(0)-\Delta u(0),
$$

such that the initial condition for $\Delta s$, which is needed in Eq. (26), is known once the initial value of $\Delta u$ has been determined from Eq. (24). The critical velocity can then be obtained from

$$
\begin{aligned}
V^{\ddagger} & =v(0)=\lambda_{\mathrm{u}} u(0)+\lambda_{\mathrm{s}} s(0) \\
& =\left(\lambda_{\mathrm{u}}-\lambda_{\mathrm{s}}\right) u(0) \quad \text { as } x(0)=u(0)+s(0)=0 \\
& =\left(\lambda_{\mathrm{u}}-\lambda_{\mathrm{s}}\right)\left[u^{\ddagger}(0)+\Delta u(0)\right] .
\end{aligned}
$$

In the harmonic approximation the trajectory that starts in the DS $x=0$ and lies in the stable manifold is given by

$$
\Delta u_{0}(t)=0 \quad \text { and } \quad \Delta s_{0}(t)=-x^{\ddagger}(0) e^{\lambda_{s} t} .
$$

For this case, Eq. (27) leads back to the result (23)

$$
V_{0}^{\ddagger}=\left(\lambda_{\mathrm{u}}-\lambda_{\mathrm{s}}\right) u^{\ddagger}(0) .
$$

When the solution (28) is substituted into the integral equations (24) or (26), the coordinate $x=x^{\ddagger}+\Delta u+\Delta s$ is 
replaced by

$$
X(t)=x^{\ddagger}(t)-e^{\lambda_{s} t} x^{\ddagger}(0) .
$$

This function represents the harmonic approximation to the coordinate $x(t)$ of the trajectory under study. Moreover, it constitutes a suitable basis of the perturbative expansion.

The leading-order correction to the critical velocity can be obtained from (24) as

$$
\Delta u_{\text {lead }}(t)=\frac{1}{\lambda_{\mathrm{u}}-\lambda_{\mathrm{s}}} S\left[\lambda_{\mathrm{u}}, f(X) ; t\right],
$$

from which it follows that

$$
V_{\text {lead }}^{\ddagger}=S\left[\lambda_{\mathrm{u}}, f(X) ; 0\right] .
$$

To obtain higher order corrections to the critical velocity in a systematic manner, we introduce the expansions

$$
\begin{aligned}
V^{\ddagger} & =V_{0}^{\ddagger}+\varepsilon V_{1}^{\ddagger}+\varepsilon^{2} V_{2}^{\ddagger}+\cdots \\
\Delta u & =\varepsilon \Delta u_{1}+\varepsilon^{2} \Delta u_{2}+\cdots \\
\Delta s & =-x^{\ddagger}+\varepsilon \Delta s_{1}+\varepsilon^{2} \Delta s_{2}+\cdots .
\end{aligned}
$$

We will write

$$
\Delta x_{k}=\Delta u_{k}+\Delta s_{k} \quad \text { for } \quad k \geq 1 .
$$

Expand the anharmonic term as

$$
f\left(X+\varepsilon \Delta x_{1}+\varepsilon^{2} \Delta x_{2}+\cdots\right)=\varepsilon f_{1}+\varepsilon^{2} f_{2}+\cdots,
$$

where terms on the r.h.s. depend on the $\Delta x_{j}$. Since $f$ is assumed to have an overall order $\varepsilon$ or higher, the calculation of the term $f_{k}$ requires only the knowledge of $\Delta x_{j}$ for $j<k$. Equations (24), (26), and (31) then yield the recurrence relations

$$
\begin{aligned}
\Delta u_{k}(t) & =\frac{1}{\lambda_{\mathrm{u}}-\lambda_{\mathrm{s}}} S\left[\lambda_{\mathrm{u}}, f_{k} ; t\right], \\
\Delta s_{k}(t) & =-\Delta u_{k}(0) e^{\lambda_{\mathrm{s}} t}-\frac{1}{\lambda_{\mathrm{u}}-\lambda_{\mathrm{s}}} \bar{S}\left[\lambda_{\mathrm{s}}, f_{k} ; t\right], \\
\Delta x_{k}(t) & =\Delta u_{k}(t)+\Delta s_{k}(t),
\end{aligned}
$$

from which it can be finally obtained

$$
V_{k}^{\ddagger}=\left(\lambda_{\mathrm{u}}-\lambda_{\mathrm{s}}\right) \Delta u_{k}(0) .
$$

The recursion relations (33) can be successively evaluated as written for $k=1,2, \ldots$ up to any desired order.

For example, for the anharmonic force corresponding to the generic one-dimensional potential (10) with only cubic and quartic terms, expansion (32) gives

$$
\begin{aligned}
& f_{1}=-c_{3} X^{2}, \\
& f_{2}=-c_{4} X^{3}-2 c_{3} X \Delta x_{1} .
\end{aligned}
$$

It is then obtained

$$
\begin{aligned}
\Delta u_{1}(t) & =-\frac{c_{3}}{\lambda_{\mathrm{u}}-\lambda_{\mathrm{s}}} S\left[\lambda_{\mathrm{u}}, X^{2} ; t\right], \\
\Delta s_{1}(t) & =\frac{c_{3}}{\lambda_{\mathrm{u}}-\lambda_{\mathrm{s}}}\left(S\left[\lambda_{\mathrm{u}}, X^{2} ; 0\right] e^{\lambda_{\mathrm{s}} t}+\bar{S}\left[\lambda_{\mathrm{s}}, X^{2} ; t\right]\right), \\
\Delta x_{1}(t) & =\frac{c_{3}}{\lambda_{\mathrm{u}}-\lambda_{\mathrm{s}}}\left(S\left[\lambda_{\mathrm{u}}, X^{2} ; 0\right] e^{\lambda_{\mathrm{s}} t}-S\left[\lambda_{\mathrm{u}}, X^{2} ; t\right]+\bar{S}\left[\lambda_{\mathrm{s}}, X^{2} ; t\right]\right),
\end{aligned}
$$

$$
\begin{aligned}
\Delta u_{2}(t)= & -\frac{1}{\lambda_{\mathrm{u}}-\lambda_{\mathrm{s}}} S\left[\lambda_{\mathrm{u}}, 2 c_{3} X \Delta x_{1}+c_{4} X^{3} ; t\right] \\
= & -\frac{c_{4}}{\lambda_{\mathrm{u}}-\lambda_{\mathrm{s}}} S\left[\lambda_{\mathrm{u}}, X^{3} ; t\right]-\frac{2 c_{3}^{2}}{\left(\lambda_{\mathrm{u}}-\lambda_{\mathrm{s}}\right)^{2}} \\
& \times S_{\tau}\left[\lambda_{\mathrm{u}}, X(\tau)\left(e^{\lambda_{\mathrm{s}} \tau} S\left[\lambda_{\mathrm{u}}, X^{2} ; 0\right]-S\left[\lambda_{\mathrm{u}}, X^{2} ; \tau\right]\right.\right. \\
& \left.\left.+\bar{S}\left[\lambda_{\mathrm{s}}, X^{2} ; \tau\right]\right) ; t\right] .
\end{aligned}
$$

From (34) we have that

$$
V_{1}^{\ddagger}=-c_{3} S\left[\lambda_{\mathrm{u}}, X^{2} ; 0\right],
$$

in agreement with Eq. (30), and

$$
\begin{aligned}
V_{2}^{\ddagger}= & -c_{4} S\left[\lambda_{\mathrm{u}}, X^{3} ; 0\right]-\frac{2 c_{3}^{2}}{\lambda_{\mathrm{u}}-\lambda_{\mathrm{s}}} S_{\tau}\left[\lambda_{\mathrm{u}}, X(\tau)\left(e^{\lambda_{\mathrm{s}} \tau} S\left[\lambda_{\mathrm{u}}, X^{2} ; 0\right]\right.\right. \\
& \left.\left.-S\left[\lambda_{\mathrm{u}}, X^{2} ; \tau\right]+\bar{S}\left[\lambda_{\mathrm{s}}, X^{2} ; \tau\right]\right) ; 0\right] .
\end{aligned}
$$

Not surprisingly, the corrections (35) and (36) depend, through the function $X$, on the realization of the noise. This dependence reflects the fact that on an anharmonic potential not only the position, but also the shape of the invariant manifolds, are stochastically time dependent.

We also calculated the critical velocity numerically for a given realization of the noise. To this end, an ensemble of trajectories starting on the DS was propagated numerically. By recording which trajectories were reactive and which were not, the value of the critical velocity could be bracketed with high accuracy. For one fixed realization and for a potential with only a cubic anharmonic term, the perturbative expansion is compared to numerical results in Fig. 2. There is good agreement between perturbative and numerical results. Similar figures are obtained for other realizations of the noise, thus leading to the same conclusion. Obviously, the size of the first and second-order corrections, as well as that of the higher order terms that are omitted, varies among different realizations.

In the special case that the anharmonic potential contains only a quartic term, the perturbation expansion results as an expansion in powers of $\varepsilon^{2}$, with the odd orders terms null. For the first two non-zero corrections, a similar calculation shows that

$$
V_{2}^{\ddagger}=-c_{4} S\left[\lambda_{\mathrm{u}}, X^{3} ; 0\right],
$$

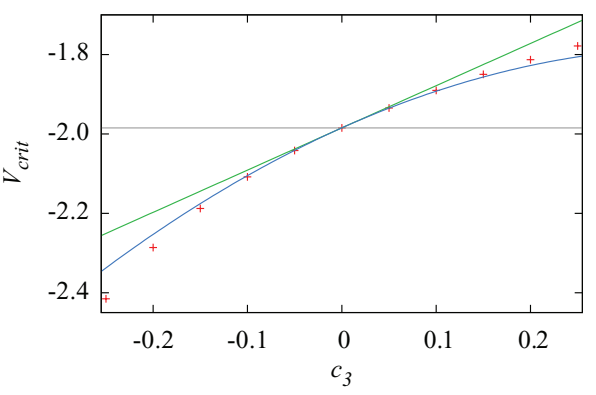

FIG. 2. Critical velocity for a realization of the noise for a one-dimensional barrier with cubic anharmonicity, $c_{3}$, for $\omega_{\mathrm{b}}=1, \gamma=2, k_{\mathrm{B}} T=1$. Numerical simulation results (red crosses), harmonic approximation (23) (gray horizontal line), perturbative results to first-order $(23)+(35)$ (green straight line) and second-order $(23)+(35)+(36)$ (blue line) 


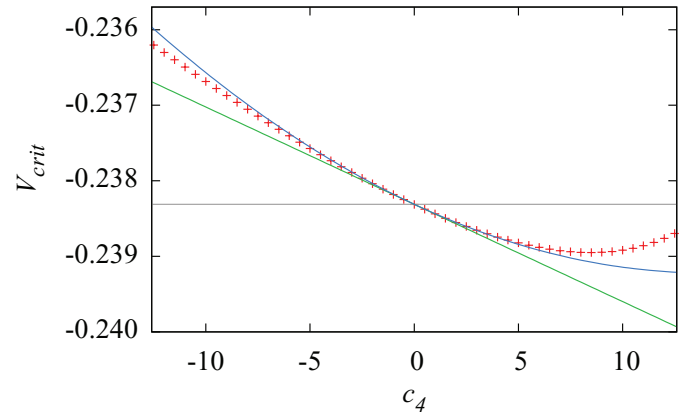

FIG. 3. Critical velocity for one realization of the noise for a onedimensional barrier with quartic anharmonicity, $c_{4}$, for $\omega_{\mathrm{b}}=1, \gamma=2.5$, $k_{\mathrm{B}} T=1$. Numerical simulation results (red crosses), harmonic approximation (23) (gray horizontal line), perturbative results to first-order (23) + (37) (green straight line) and second-order $(23)+(37)+(38)$ (blue line).

which is again consistent with Eq. (30), and

$$
\begin{aligned}
V_{4}^{\ddagger}= & -\frac{3 c_{4}^{2}}{\lambda_{\mathrm{u}}-\lambda_{\mathrm{s}}} S_{\tau}\left[\lambda_{\mathrm{u}}, X^{2}(\tau)\left(e^{\lambda_{\mathrm{s}} \tau} S\left[\lambda_{\mathrm{u}}, X^{3} ; 0\right]-S\left[\lambda_{\mathrm{u}}, X^{3} ; \tau\right]\right.\right. \\
& \left.\left.+\bar{S}\left[\lambda_{\mathrm{s}}, X^{3} ; \tau\right]\right) ; 0\right] .
\end{aligned}
$$

A comparison of the perturbative corrections (37) and (38) with numerical results is shown in Fig. 3. Again, this comparison confirms the accuracy of the perturbative results.

The function $X$ introduced in Eq. (29) plays a special role in the perturbation expansion because it represents the unperturbed trajectory. To obtain a different perspective of this function, note that the critical velocity should depend only on the behavior of the stochastic force $\xi_{\alpha}(t)$ for $t \geq 0$, but not on the driving at earlier times: Once the initial conditions of a trajectory at $t=0$ are given, its future fate can only depend on the future noise. The separatrix between reactive and nonreactive trajectories must therefore also be determined by only the future noise. Yet the perturbation term in (21) depends, via $x^{\ddagger}(t)$, on $s^{\ddagger}(t)$, which is given by past noise.

If we split up the integration range of the $S$ functional, we find that for $t \geq 0$

$$
s^{\ddagger}(t)=e^{\lambda_{\mathrm{s}} t} s^{\ddagger}(0)+\int_{0}^{t} e^{\lambda_{\mathrm{s}}(t-\tau)} \xi_{\alpha}(\tau) d \tau .
$$

The integral in this expression depends only on noise for $t \geq 0$. The term including $s^{\ddagger}(0)$ contains all the dependence on the past, and it drops out when we form $X(t)$. The variable $X$ is the simplest modification of $x^{\ddagger}$ in which the dependence on the past has been removed.

\section{CORRECTIONS TO THE REACTION RATES}

\section{A. General rate expressions}

In a one-dimensional model, the characteristic function $\chi_{\mathrm{r}}$ can be expressed in terms of the critical velocity as

$$
\chi_{\mathrm{r}}\left(v_{x}\right)=\left\{\begin{array}{lll}
1 & : & v_{x}>V^{\ddagger}, \\
0 & : & v_{x}<V^{\ddagger} .
\end{array}\right.
$$

In contrast to the TST approximation (5), and in spite of its simplicity, the expression (39) is exact. It allows to evaluate the average over initial conditions in Eq. (7) — the factor $p_{\perp}$ in
Eq. (3) being absent in one dimension — to find

$$
\kappa=\left\langle\exp \left(-\frac{V^{\ddagger 2}}{2 k_{\mathrm{B}} T}\right)\right\rangle_{\alpha},
$$

where only the average over the noise remains. This expression was derived in Ref. 33 for a harmonic barrier. It is now clear that the same expression holds also for anharmonic potentials if the critical velocity $V^{\ddagger}$ is suitably modified. Remarkably, no anharmonic corrections arise in the rate expression (40).

If we have a perturbative expansion

$$
V^{\ddagger}=V_{0}^{\ddagger}+\varepsilon V_{1}^{\ddagger}+\varepsilon^{2} V_{2}^{\ddagger}+\cdots,
$$

we can substitute into (40) and expand the exponential to obtain a series of rate corrections

$$
\kappa=\kappa_{0}+\varepsilon \kappa_{1}+\varepsilon^{2} \kappa_{2}+\cdots,
$$

where

$$
\begin{gathered}
\kappa_{0}=\langle P\rangle_{\alpha}, \\
\kappa_{1}=-\frac{1}{k_{\mathrm{B}} T}\left\langle P V_{0}^{\ddagger} V_{1}^{\ddagger}\right\rangle_{\alpha}, \\
\kappa_{2}=\frac{1}{2\left(k_{\mathrm{B}} T\right)^{2}}\left\langle P V_{0}^{\ddagger 2} V_{1}^{\ddagger 2}\right\rangle_{\alpha}-\frac{1}{k_{\mathrm{B}} T}\left\langle P V_{0}^{\ddagger} V_{2}^{\ddagger}\right\rangle_{\alpha} \\
-\frac{1}{2 k_{\mathrm{B}} T}\left\langle P V_{1}^{\ddagger 2}\right\rangle_{\alpha}
\end{gathered}
$$

with the abbreviation

$$
P=\exp \left(-\frac{V_{0}^{\ddagger 2}}{2 k_{\mathrm{B}} T}\right)=\exp \left(-\frac{\left(\lambda_{\mathrm{u}}-\lambda_{\mathrm{s}}\right)^{2} u^{\ddagger 2}(0)}{2 k_{\mathrm{B}} T}\right) .
$$

We will now address the problem of evaluating the noise averages in Eq. (43).

\section{B. Distorted correlation functions}

The corrections to the critical velocity that appear in the averages (43) are expressed in terms of the function $X(t)$, which is in turn given in terms of the components $u^{\ddagger}(t)$ and $s^{\ddagger}(t)$ of the TS trajectory. They are Gaussian random variables whose correlation functions were evaluated in Ref. 31. In the current notation and with

$$
\sigma^{2}=\frac{k_{\mathrm{B}} T \gamma}{\left|\lambda_{\mathrm{s}}\right|\left(\lambda_{\mathrm{u}}-\lambda_{\mathrm{s}}\right)^{2}}
$$

they read, for $t \geq 0$, as

$$
\left\langle s^{\ddagger}(t) s^{\ddagger}(0)\right\rangle_{\alpha}=\sigma^{2} e^{\lambda_{s} t},
$$

$$
\begin{gathered}
\left\langle u^{\ddagger}(t) u^{\ddagger}(0)\right\rangle_{\alpha}=-\frac{\lambda_{\mathrm{s}}}{\lambda_{\mathrm{u}}} \sigma^{2} e^{-\lambda_{\mathrm{u}} t}, \\
\left\langle u^{\ddagger}(t) s^{\ddagger}(0)\right\rangle_{\alpha}=0,
\end{gathered}
$$

$$
\left\langle s^{\ddagger}(t) u^{\ddagger}(0)\right\rangle_{\alpha}=\frac{2 \lambda_{\mathrm{s}}}{\lambda_{\mathrm{u}}+\lambda_{\mathrm{s}}} \sigma^{2}\left(e^{-\lambda_{\mathrm{u}} t}-e^{\lambda_{\mathrm{s}} t}\right) .
$$


To evaluate the corrections (43) to the reaction rate, we need to calculate noise averages of the form $\langle P(\ldots)\rangle_{\alpha}$, where $(\ldots)$ indicates some expression in the functions $u^{\ddagger}(t)$ and $s^{\ddagger}(t)$. We will therefore assume that the expression (...) can be written as a function of finitely many random variables $z=\left(z_{1}, \ldots, z_{n}\right)$ that follow a multidimensional Gaussian distribution with zero mean and covariance matrix $\Sigma$, i.e., the matrix elements of $\Sigma$ are $\sigma_{i j}=\left\langle z_{i} z_{j}\right\rangle_{\alpha}$. As the first component we include the variable $z_{1}=u^{\ddagger}(0)$, which plays a special role because it occurs in the factor $P$ in Eq. (44).

Using (23) and setting $\rho=\left(\lambda_{\mathrm{u}}-\lambda_{\mathrm{S}}\right)^{2} / k_{\mathrm{B}} T$, we can write

$$
\begin{aligned}
\langle P(\ldots)\rangle_{\alpha} & =\frac{1}{\sqrt{(2 \pi)^{n} \operatorname{det} \Sigma}} \int d^{n} z e^{-z^{\mathrm{T}} \Sigma^{-1} z / 2} e^{-\rho z_{1}^{2} / 2}(\ldots) \\
& =\frac{1}{\sqrt{(2 \pi)^{n} \operatorname{det} \Sigma}} \int d^{n} z e^{-z^{\mathrm{T}}\left(\Sigma^{-1}+\rho J\right) z / 2}(\ldots) \\
& =\sqrt{\frac{\operatorname{det} \Sigma_{0}}{\operatorname{det} \Sigma}} \frac{1}{\sqrt{(2 \pi)^{n} \operatorname{det} \Sigma_{0}}} \int d^{n} z e^{-z^{\mathrm{T}} \Sigma_{0}^{-1} z / 2}(\ldots), \\
& =\sqrt{\frac{\operatorname{det} \Sigma_{0}}{\operatorname{det} \Sigma}}\langle\ldots\rangle_{0},
\end{aligned}
$$

where we have introduced the matrix

$$
J=\left(\begin{array}{cccc}
1 & 0 & 0 & \ldots \\
0 & 0 & 0 & \ldots \\
0 & 0 & 0 & \ldots \\
\vdots & \vdots & \vdots & \ddots
\end{array}\right)
$$

and we have used $\langle\ldots\rangle_{0}$ to denote an average over a multidimensional Gaussian distribution with the modified covariance matrix $\Sigma_{0}$ given by

$$
\Sigma_{0}^{-1}=\Sigma^{-1}+\rho J .
$$

From the observation

$$
\Sigma J=\left(\begin{array}{cccc}
\sigma_{11} & 0 & \ldots & 0 \\
\sigma_{21} & 0 & & 0 \\
\vdots & & \ddots & \vdots \\
\sigma_{n 1} & 0 & \ldots & 0
\end{array}\right)
$$

we obtain $(\Sigma J)^{2}=\sigma_{11} \Sigma J$. It is then easy to check that

$$
\left(\Sigma-\frac{\rho}{1+\rho \sigma_{11}} \Sigma J \Sigma\right)\left(\Sigma^{-1}+\rho J\right)=I,
$$

the identity matrix. Therefore,

$$
\begin{aligned}
\Sigma_{0} & =\Sigma-\frac{\rho}{1+\rho \sigma_{11}} \Sigma J \Sigma \\
& =\Sigma+\frac{\lambda_{\mathrm{u}}}{\lambda_{\mathrm{s}}} \rho \Sigma J \Sigma,
\end{aligned}
$$

where in the last step we have used the value given in Eq. (46) for $\sigma_{11}=\left\langle u^{\ddagger 2}(0)\right\rangle_{\alpha}$.

Furthermore,

$$
\Sigma_{0} \Sigma^{-1}=I-\frac{\rho}{1+\rho \sigma_{11}} \Sigma J
$$

is a lower triangular matrix whose diagonal elements, except for the $(1,1)$ element, are all equal to 1 . This observation makes it easy to evaluate

$$
\begin{aligned}
\frac{\operatorname{det} \Sigma_{0}}{\operatorname{det} \Sigma} & =\operatorname{det}\left(I-\frac{\rho}{1+\rho \sigma_{11}} \Sigma J\right) \\
& =1-\frac{\rho \sigma_{11}}{1+\rho \sigma_{11}} \\
& =-\frac{\lambda_{\mathrm{u}}}{\lambda_{\mathrm{s}}}=\frac{\lambda_{\mathrm{u}}^{2}}{\omega_{\mathrm{b}}^{2}}
\end{aligned}
$$

where Eq. (46) has again be used.

Substituting Eq. (49) in Eq. (47), we finally find

$$
\langle P(\ldots)\rangle_{\alpha}=\frac{\lambda_{\mathrm{u}}}{\omega_{\mathrm{b}}}\langle\ldots\rangle_{0} .
$$

For the components of the modified covariance matrix (48) we find

$$
\left\langle z_{i} z_{j}\right\rangle_{0}=\left\langle z_{i} z_{j}\right\rangle_{\alpha}+\frac{\lambda_{\mathrm{u}}}{\lambda_{\mathrm{s}}} \rho\left\langle u^{\ddagger}(0) z_{i}\right\rangle_{\alpha}\left\langle u^{\ddagger}(0) z_{j}\right\rangle_{\alpha},
$$

which allows to obtain the moments of the distorted Gaussian distribution once the moments of the original Gaussian are known. In particular, $\left\langle z_{i} z_{j}\right\rangle_{0}=\left\langle z_{i} z_{j}\right\rangle_{\alpha}$ if either $z_{i}$ or $z_{j}$ are uncorrelated with $u^{\ddagger}(0)$.

Once the second moments of the distorted Gaussian distribution, i.e., the matrix elements of $\Sigma_{0}$, are known, Isserlis' theorem $^{43,44}$ can be used to express higher order moments in terms of second moments, e.g.,

$$
\begin{aligned}
& \left\langle z_{1} z_{2} z_{3} z_{4}\right\rangle_{0} \\
& \quad=\left\langle z_{1} z_{2}\right\rangle_{0}\left\langle z_{3} z_{4}\right\rangle_{0}+\left\langle z_{1} z_{3}\right\rangle_{0}\left\langle z_{2} z_{4}\right\rangle_{0}+\left\langle z_{1} z_{4}\right\rangle_{0}\left\langle z_{2} z_{3}\right\rangle_{0} .
\end{aligned}
$$

This expression contains a sum over all possible pairings of the four factors. Other even-order moments can be evaluated in a similar way, and the odd-order moments are zero. In this way, the modified averages of arbitrary polynomials can be calculated.

The moments that will be required in the rate calculation can be obtained from these results; they are

$$
\frac{\left\langle u^{\ddagger}(0) X(t)\right\rangle_{0}}{\sigma^{2}}=\left(1-\beta_{\mathrm{u}}\right)\left(e^{-\lambda_{\mathrm{u}} t}-e^{\lambda_{\mathrm{s}} t}\right),
$$

$$
\begin{aligned}
\frac{\left\langle X(t) X\left(t^{\prime}\right)\right\rangle_{0}}{\sigma^{2}}= & \left(1-\beta_{\mathrm{s}}\right) e^{\lambda_{\mathrm{s}}\left|t-t^{\prime}\right|}-\frac{\lambda_{\mathrm{s}}}{\lambda_{\mathrm{u}}}\left(1-\beta_{\mathrm{u}}\right) e^{-\lambda_{\mathrm{u}}\left|t-t^{\prime}\right|} \\
& +\left(1-2 \beta_{\mathrm{s}}+\frac{\lambda_{\mathrm{s}}}{\lambda_{\mathrm{u}}}\right) e^{-\lambda_{\mathrm{u}}\left(t+t^{\prime}\right)} \\
& +\left(1-\beta_{\mathrm{u}}\right)\left(e^{-\lambda_{\mathrm{u}} t+\lambda_{\mathrm{s}} t^{\prime}}+e^{-\lambda_{\mathrm{u}} t^{\prime}+\lambda_{\mathrm{s}} t}\right),
\end{aligned}
$$

with

$$
\beta_{\mathrm{u}}=\frac{2 \lambda_{\mathrm{u}}}{\lambda_{\mathrm{u}}+\lambda_{\mathrm{s}}}, \quad \beta_{\mathrm{s}}=\frac{2 \lambda_{\mathrm{s}}}{\lambda_{\mathrm{u}}+\lambda_{\mathrm{s}}} .
$$




\section{Results for the one-dimensional potential}

With the help of Eq. (50) the leading term in the transmission factor (43a) can be evaluated, giving

$$
\kappa_{0}=\frac{\lambda_{\mathrm{u}}}{\omega_{\mathrm{b}}} .
$$

This is the famous Kramers result for the transmission factor. ${ }^{17}$

The perturbation expansion is set up in such a way that effectively the noise carries a factor of $\varepsilon$. The critical velocity $V_{0}^{\ddagger}$ is linear in the noise. If $V_{1}^{\ddagger}$ is one order $\varepsilon$ higher, it must be quadratic in the noise, and $V_{2}^{\ddagger}$ cubic. Consequently,

$$
\kappa_{1}=-\frac{1}{k_{\mathrm{B}} T} \frac{\lambda_{\mathrm{u}}}{\omega_{\mathrm{b}}}\left\langle V_{0}^{\ddagger} V_{1}^{\ddagger}\right\rangle_{0}=0
$$

is a third-order moment of the noise and must vanish. Similarly, all odd-order corrections to the transmission factor must be zero. According to the fluctuation-dissipation theorem (9), the noise carries a factor $\sqrt{k_{\mathrm{B}} T}$, so that a perturbative expansion in powers of $\varepsilon$ corresponds to an expansion in powers of $\sqrt{k_{\mathrm{B}} T}$. By contrast, Eq. (42) is an expansion of the transmission factor in powers of $k_{\mathrm{B}} T$ because it has only even-order terms.

The simplest rate correction can therefore be obtained from a quartic perturbation in the potential. We set $c_{3}=0$, which makes $V_{1}^{\ddagger}=0$, and calculate the rate correction that is linear in $c_{4}$. Substituting Eqs. (23) and (37) into (43b), it is found that

$$
\kappa_{2}^{c_{4}}=\frac{c_{4}\left(\lambda_{\mathrm{u}}-\lambda_{\mathrm{s}}\right)}{k_{\mathrm{B}} T} S_{\tau}\left[\lambda_{\mathrm{u}},\left\langle P u^{\ddagger}(0) X^{3}(\tau)\right\rangle_{\alpha} ; 0\right] .
$$

The average over the noise can be brought inside the $S$ functional because the latter is shorthand notation for an integral. The remaining moment can be evaluated as

$$
\begin{aligned}
\left\langle P u^{\ddagger}(0) X^{3}(\tau)\right\rangle_{\alpha} & =\frac{\lambda_{\mathrm{u}}}{\omega_{\mathrm{b}}}\left\langle u^{\ddagger}(0) X^{3}(\tau)\right\rangle_{0} \\
& =3 \frac{\lambda_{\mathrm{u}}}{\omega_{\mathrm{b}}}\left\langle u^{\ddagger}(0) X(\tau)\right\rangle_{0}\left\langle X^{2}(\tau)\right\rangle_{0} .
\end{aligned}
$$

The modified correlation functions that are required here are given in Eq. (52). Equation (55) can thus be rewritten as a sum of exponentially decaying terms, for which the $S$ functional in Eq. (54) is easy to evaluate. This procedure yields

$$
\kappa_{2}^{c_{4}}=-\frac{3 c_{4} \sigma^{4}\left(\lambda_{\mathrm{u}}-\lambda_{\mathrm{s}}\right)^{2}}{4 k_{\mathrm{B}} T \omega_{\mathrm{b}} \lambda_{\mathrm{u}}}=\frac{3}{4} c_{4} k_{\mathrm{B}} T \frac{\gamma^{2}}{\omega_{\mathrm{b}}^{3} \lambda_{\mathrm{s}}\left(\lambda_{\mathrm{u}}-\lambda_{\mathrm{s}}\right)^{2}} .
$$

This result agrees with the perturbative correction given in Refs. 26-28. It can be rewritten as

$$
\frac{\kappa_{2}^{c_{4}}}{\kappa_{0}}=-\frac{3}{4} \frac{c_{4} k_{\mathrm{B}} T}{\omega_{\mathrm{b}}^{4}}\left(\frac{1-\mu^{2}}{1+\mu^{2}}\right)^{2}
$$

in terms of the dimensionless parameter $\mu=\kappa_{0}=\lambda_{\mathrm{u}} / \omega_{\mathrm{b}}$ that was used in Ref. 27.

A comparison of Eq. (56) with numerical results is shown in Fig. 4. They confirm once more that the perturbative result is correct. The figure also shows the second-order correction
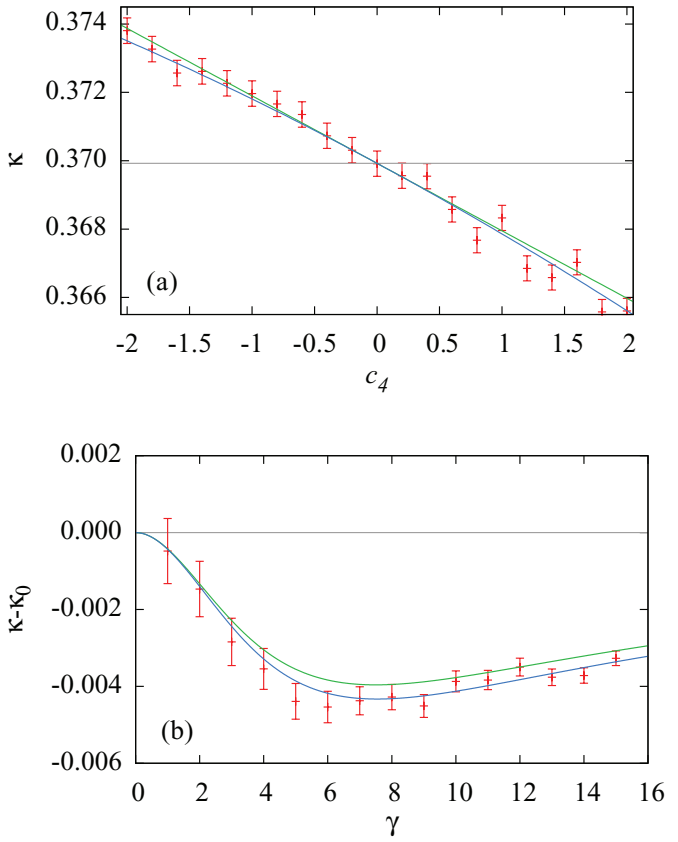

FIG. 4. Transmission factor, $\kappa$, for a one-dimensional potential with quartic anharmonicity, $c_{4}$, for $\omega_{\mathrm{b}}=3, k_{\mathrm{B}} T=1$. (a) $\kappa$ as a function of the coupling strength $c_{4}$ for a value of the damping $\gamma=7$. (b) Difference between $\kappa$ and its Kramers approximation, $\kappa_{0}$, as a function of $\gamma$ for $c_{4}=2$. Numerical simulation results (red points), harmonic (Kramers) approximation (53) (gray horizontal line), perturbative results to first-order, obtained from $(53)+(56)$ (green line), and second-order obtained from $(53)+(56)+(58)$ (blue line).

in $c_{4}$, which can be obtained in a similar way from Eq. (38). It reads

$$
\begin{aligned}
\frac{\kappa_{4}^{c_{4}}}{\kappa_{0}}= & -\frac{3}{32}\left(\frac{c_{4} k_{\mathrm{B}} T}{\omega_{\mathrm{b}}^{4}}\right)^{2}\left(\frac{1-\mu^{2}}{1+\mu^{2}}\right)^{4} \\
& \times \frac{105 \mu^{8}+830 \mu^{6}+1648 \mu^{4}+770 \mu^{2}+87}{\left(1-\mu^{4}\right)\left(3 \mu^{4}+10 \mu^{2}+3\right)} .
\end{aligned}
$$

In the numerical example the second-order contribution is small, but Fig. 4(b) shows clearly that the second-order perturbative result is in better agreement with the numerical data than the first-order result.

For a generic anharmonic potential that has a third-order term, the leading rate correction is quadratic in $c_{3}$ and can be obtained from Eq. (43c) with the help of Eqs. (35) and (36). It reads

$$
\frac{\kappa_{2}^{c_{3}}}{\kappa_{0}}=-\frac{1}{6} \frac{c_{3}^{2} k_{\mathrm{B}} T}{\omega_{\mathrm{b}}^{6}}\left(\frac{1-\mu^{2}}{1+\mu^{2}}\right)^{2} \frac{10 \mu^{4}+41 \mu^{2}+10}{2 \mu^{4}+5 \mu^{2}+2} .
$$

A comparison between Eq. (59) and numerical data is shown in Fig. 5. Again, the agreement is excellent.

If both cubic and quartic perturbations are present in the potential, then the second-order contribution to the Kramers' transmission factor is equal to the sum of expressions (57) and (59). 


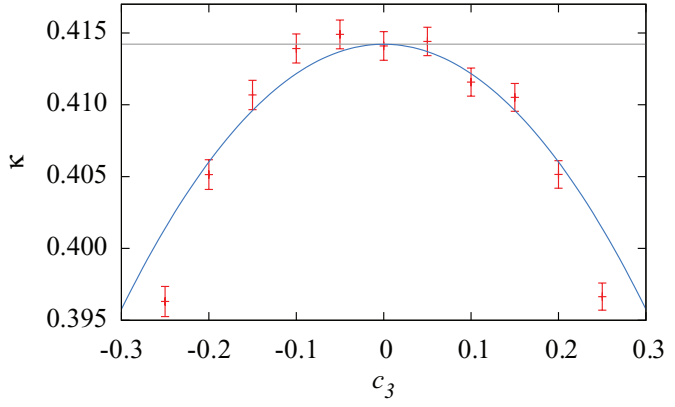

FIG. 5. Transmission factor for a one-dimensional potential with cubic anharmonicity, $c_{3}$, with $\omega_{\mathrm{b}}=1, \gamma=2, k_{\mathrm{B}} T=1$. Numerical simulation results (red points), harmonic (Kramers) approximation (53) (gray horizontal line), perturbative results to second-order, obtained from (53) + (59) (blue line). Notice that in this case the first-order correction is zero.

\section{THE TWO-DIMENSIONAL CASE}

So far, our discussion of the stochastic stable and unstable manifolds and their use has been restricted to a onedimensional model. Most problems of physical interest, however, have several degrees of freedom. It is therefore crucial to show how the results obtained before can be generalized to higher dimension. We will carry out the generalization to two dimensions, which requires some extensions of the previous discussion. It will then be obvious that these techniques can equally be applied to systems in arbitrary dimension.

We study a two-dimensional model whose dynamics is described by the Langevin equation (8). We denote the configuration space coordinates as $\boldsymbol{q}=(x, y)$ and the corresponding velocities as $\dot{\boldsymbol{q}}=\left(v_{x}, v_{y}\right)$. The friction matrix $\boldsymbol{\Gamma}=\gamma I_{2}$ is assumed to be a scalar multiple of the $2 \times 2$ identity matrix, $I_{2}$. By the fluctuation-dissipation theorem (9), this assumption implies that the $x$ and $y$ components of the fluctuating force are statistically uncorrelated. For demonstration purposes we will use the anharmonic model potential

$$
U(x, y)=-\frac{1}{2} \omega_{\mathrm{b}}^{2} x^{2}+\frac{1}{2} \omega_{y}^{2} y^{2}+c x^{2} y^{2}
$$

that has already been used in Refs. 32 and 33. The anharmonic perturbation in Eq. (60) is of fourth order. In the terminology of the previous sections, the coupling parameter $c$ is therefore of order $\varepsilon^{2}$, and rate corrections at first order in $c$ are expected.

\section{A. Invariant manifolds in higher dimension}

In a two-dimensional setting, the phase space of the Langevin equation (8) is four-dimensional. It can be described with coordinates $\left(x, y, v_{x}, v_{y}\right)$. As before, the harmonic approximation of the dynamics around the barrier can be diagonalized by introducing the coordinates $u$ and $s$ given in Eq. (12) and coordinates $z_{1}$ and $z_{2}$ defined by

$$
z_{1}=\frac{v_{y}-\lambda_{2} y}{\lambda_{1}-\lambda_{2}}, \quad z_{2}=\frac{v_{y}-\lambda_{1} y}{\lambda_{2}-\lambda_{1}}
$$

with the inverse transformation

$$
y=z_{1}+z_{2}, \quad v_{y}=\lambda_{1} z_{1}+\lambda_{2} z_{2} .
$$

The two additional eigenvalues

$$
\lambda_{1,2}=-\frac{1}{2}\left(\gamma \pm \sqrt{\gamma^{2}-4 \omega_{y}^{2}}\right)
$$

are either real and negative or form a pair of complex conjugates with negative real parts.

The fluctuating force has two independent components $\xi_{x, \alpha}(t)$ and $\xi_{y, \alpha}(t)$, which determine the four components of the TS trajectory

$$
\begin{aligned}
u^{\ddagger}(t) & =\frac{1}{\lambda_{\mathrm{u}}-\lambda_{\mathrm{s}}} S\left[\lambda_{\mathrm{u}}, \xi_{x, \alpha} ; t\right], \\
s^{\ddagger}(t) & =-\frac{1}{\lambda_{\mathrm{u}}-\lambda_{\mathrm{s}}} S\left[\lambda_{\mathrm{s}}, \xi_{x, \alpha} ; t\right], \\
z_{1}^{\ddagger}(t) & =\frac{1}{\lambda_{1}-\lambda_{2}} S\left[\lambda_{1}, \xi_{y, \alpha} ; t\right], \\
z_{2}^{\ddagger}(t) & =-\frac{1}{\lambda_{1}-\lambda_{2}} S\left[\lambda_{2}, \xi_{y, \alpha} ; t\right]
\end{aligned}
$$

that serves as a time-dependent coordinate origin. In the relative coordinates

$$
\begin{gathered}
\Delta u=u-u^{\ddagger}, \quad \Delta s=s-s^{\ddagger}, \\
\Delta z_{1}=z_{1}-z_{1}^{\ddagger}, \quad \Delta z_{2}=z_{2}-z_{2}^{\ddagger}
\end{gathered}
$$

the Langevin equation is written as

$$
\begin{gathered}
\Delta \dot{u}=\lambda_{\mathrm{u}} \Delta u+\frac{f_{x}(x, y)}{\lambda_{\mathrm{u}}-\lambda_{\mathrm{s}}}, \\
\Delta \dot{s}=\lambda_{\mathrm{s}} \Delta s-\frac{f_{x}(x, y)}{\lambda_{\mathrm{u}}-\lambda_{\mathrm{s}}}, \\
\Delta \dot{z}_{1}=\lambda_{1} \Delta z_{1}+\frac{f_{y}(x, y)}{\lambda_{1}-\lambda_{2}}, \\
\Delta \dot{z}_{2}=\lambda_{2} \Delta z_{2}-\frac{f_{y}(x, y)}{\lambda_{1}-\lambda_{2}},
\end{gathered}
$$

where $f_{x}$ and $f_{y}$ denote the anharmonic parts of the mean force:

$$
\begin{aligned}
& -\frac{\partial U}{\partial x}=\omega_{\mathrm{b}}^{2} x+f_{x}(x, y), \\
& -\frac{\partial U}{\partial y}=-\omega_{y}^{2} y+f_{y}(x, y) .
\end{aligned}
$$

The differential equations (66) are coupled by the conditions

$$
\begin{aligned}
& x=x^{\ddagger}+\Delta u+\Delta s, \\
& y=y^{\ddagger}+\Delta z_{1}+\Delta z_{2} .
\end{aligned}
$$

As in the one-dimensional case, the equations of motion (66) decouple and become time-independent in the harmonic limit, $f_{x}=f_{y}=0$, and the relevant phase space structures can easily be described in this case. Among the eigenvalues in Eq. (66), $\lambda_{u}$ is positive, while the other three have negative real parts. Consequently, the TS trajectory has a onedimensional unstable manifold and a three-dimensional stable manifold. The stable manifold separates reactive from nonreactive regions of phase space. The dimension of the unstable manifold, by contrast, is too low to separate distinct regions in the four-dimensional phase space. The invariant manifolds 
cannot therefore be used to distinguish trajectories with different behaviors in the remote past, but the stable manifold can be used to predict the fate of a trajectory in the future. Thus, in arbitrary dimension the invariant manifolds provide precisely the diagnostic capabilities that are needed for rate calculations.

We are particularly interested in trajectories that start on the DS $x=0$. This is a three-dimensional surface with coordinates $\left(v_{x}, y, v_{y}\right)$, embedded in the four-dimensional phase space. It intersects the three-dimensional stable manifold in a two-dimensional surface that separates reactive from nonreactive trajectories within the DS. We will call that twodimensional surface the separatrix, and it depends on the realization of the noise.

On physical grounds, we expect a trajectory to be reactive if its initial velocity $v_{x}$ is sufficiently high. The critical velocity $V^{\ddagger}$ that separates reactive from non-reactive trajectories depends, in general, on the transverse coordinates $y$ and $v_{y}$. In the harmonic limit, the critical velocity is given by (23) and is independent of these transverse coordinates. The separatrix $v_{x}=V^{\ddagger}$ is therefore a plane within the DS that is parallel to the $y-v_{y}$ plane. When anharmonicities are taken into account, the separatrix is deformed from this plane in a stochastically time-dependent way, as indicated schematically in Fig. 6(a). Nevertheless, we will still be able to describe the separatrix by specifying a critical velocity that depends on the transverse coordinates. In Sec. VI B a perturbative expansion for the function $V^{\ddagger}\left(y, v_{y}\right)$ will be developed.

It is instructive to study the actual shape of the separatrix in a representative example. Figure 7 shows the critical velocity as a function of transverse coordinates for one real-
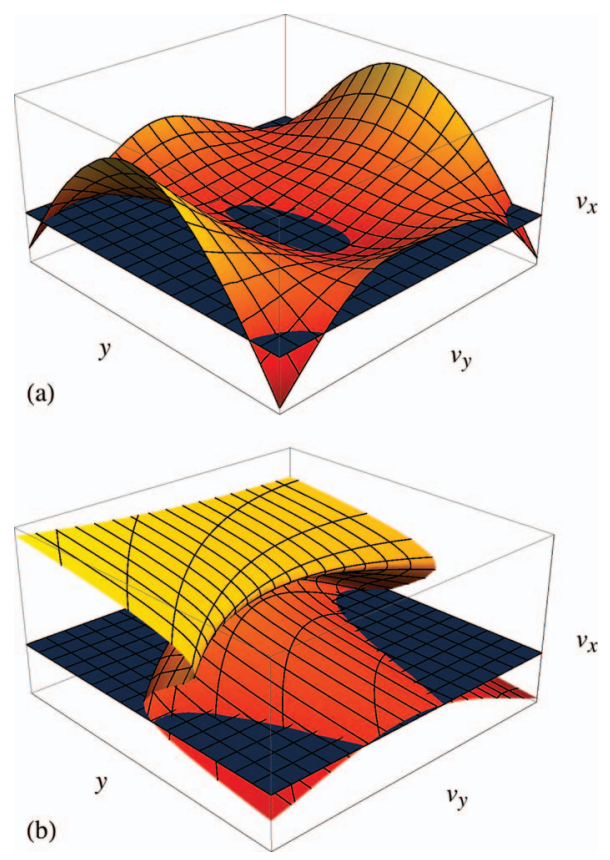

FIG. 6. Schematic representation of the separatrix within the dividing surface $x=0$. For a harmonic barrier the separatrix is a plane (gray in both panels). (a) For a weakly anharmonic barrier the separatrix can be parameterized by a function $V^{\ddagger}\left(y, v_{y}\right)$. Trajectories with initial condition $v_{x}>V^{\ddagger}\left(y, v_{y}\right)$ are reactive. (b) If anharmonicities are strong, the separatrix cannot be described by a single critical velocity, $V^{\ddagger}$.

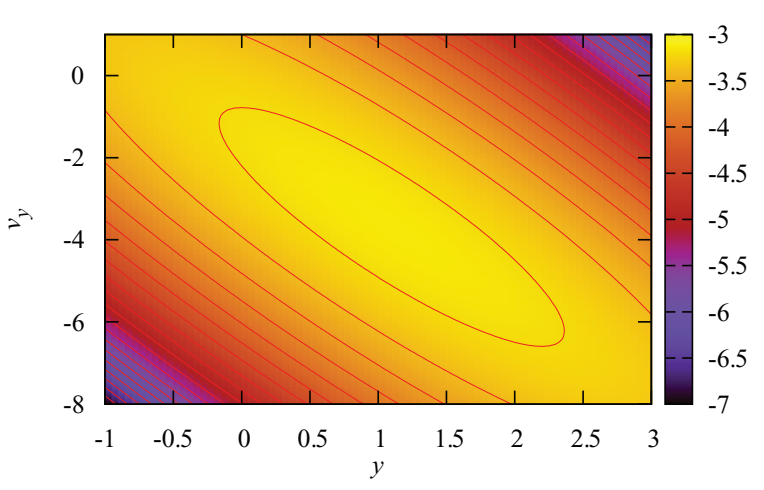

FIG. 7. Critical velocity as a function of the transverse coordinates for one realization of the noise for the two-dimensional model potential (60) for $\omega_{x}=1, \omega_{y}=1.5, \gamma=2, c=0.2, k_{\mathrm{B}} T=1$. Contour spacing is 0.2 and the central contour value is -3.2 .

ization of the noise for the two-dimensional model potential (60). The critical velocity takes a maximum that is noticeably displaced from the origin $y=v_{y}=0$. At the maximum, the critical velocity is closest to its harmonic value, which in this case is approximately -3.01 . For all values of the transverse coordinates, the critical velocity is below the harmonic approximation value. Moreover, it decays steeply away from the maximum, so that deviations from the harmonic approximation are large for most values of the coordinates. As the critical velocity appears in the exponent in the rate formula (40)—which will be generalized to higher dimension in Eq. (73) - it is expected that anharmonic effects on the critical velocity leads to large rate corrections.

If the barrier is strongly anharmonic it cannot be guaranteed, in general, that the separatrix can be parameterized by the transverse coordinates $y$ and $v_{y}$. In a situation as that indicated in Fig. 6(b), the separatrix is described by a multivalued function of the transverse coordinates. It cannot be characterized by a single critical velocity. As expected, trajectories at low $v_{x}$ are nonreactive, and those at somewhat larger $v_{x}$ are reactive. However, at certain values of $y$ and $v_{y}$, there is an interval at yet higher $v_{x}$ that also contains nonreactive trajectories. A scenario like this obviously requires very strong anharmonic effects, and this can only be achieved for large values of the transverse coordinates. But at these conditions, it is doubtful whether a TST-like treatment with a single rate-determining saddle point is appropriate at all. We will therefore neglect this possibility and assume the existence of a single critical velocity.

\section{B. Determination of the stable manifold}

As a basis for the perturbative expansion, we formally solve the differential equations (66) in terms of $S$ functionals by

$$
\begin{aligned}
\Delta u(t) & =\frac{1}{\lambda_{\mathrm{u}}-\lambda_{\mathrm{s}}} S\left[\lambda_{\mathrm{u}}, f_{x}(x, y) ; t\right], \\
\Delta s(t) & =\Delta s(0) e^{\lambda_{\mathrm{s}} t}-\frac{1}{\lambda_{\mathrm{u}}-\lambda_{\mathrm{s}}} \bar{S}\left[\lambda_{\mathrm{s}}, f_{x}(x, y) ; t\right], \\
\Delta z_{1}(t) & =\Delta z_{1}(0) e^{\lambda_{1} t}+\frac{1}{\lambda_{1}-\lambda_{2}} \bar{S}\left[\lambda_{1}, f_{y}(x, y) ; t\right],
\end{aligned}
$$




$$
\Delta z_{2}(t)=\Delta z_{2}(0) e^{\lambda_{2} t}-\frac{1}{\lambda_{1}-\lambda_{2}} \bar{S}\left[\lambda_{2}, f_{y}(x, y) ; t\right] .
$$

These integral equations are entirely analogous to Eqs. (24) and (26), and they are coupled by

$$
\begin{aligned}
& x=x^{\ddagger}+\Delta u+\Delta s, \\
& y=y^{\ddagger}+\Delta z_{1}+\Delta z_{2} .
\end{aligned}
$$

A trajectory satisfying (67) automatically lies on the stable manifold. To find the critical velocity, Eqs. (67) need to be solved under the condition that the trajectory starts in the DS $x=0$ and at the prescribed transverse coordinates $y(0)$ and $v_{y}(0)$.

We will solve Eqs. (67) by an iterative procedure as in (33). As before, the initial condition $\Delta s(0)$ must be adapted in every step in order to enforce the condition $x(0)=0$. By contrast, the transverse initial conditions $\Delta z_{1}(0)$ and $\Delta z_{2}(0)$ are fixed once and for all by imposing the condition that

$$
\begin{gathered}
y(0)=y^{\ddagger}(0)+\Delta z_{1}(0)+\Delta z_{2}(0), \\
v_{y}(0)=v_{y}^{\ddagger}(0)+\lambda_{1} \Delta z_{1}(0)+\lambda_{2} \Delta z_{2}(0)
\end{gathered}
$$

take the desired values. The critical velocity is finally obtained from Eq. (27).

Our perturbation expansion is centered around the harmonic approximation to a trajectory on the stable manifold, given by Eq. (29)

$$
X(t)=x^{\ddagger}(t)-x^{\ddagger}(0) e^{\lambda_{s} t}
$$

and

$$
Y(t)=y^{\ddagger}(t)+\Delta z_{1}(0) e^{\lambda_{1} t}+\Delta z_{2}(0) e^{\lambda_{2} t} .
$$

The latter can be split according to

$$
Y(t)=Y_{\alpha}(t)+Y_{\perp}(t)
$$

into one part

$$
Y_{\alpha}(t)=y^{\ddagger}(t)-z_{1}^{\ddagger}(0) e^{\lambda_{1} t}-z_{2}^{\ddagger}(0) e^{\lambda_{2} t}
$$

that depends on the realization of the noise but not on the initial conditions, and another

$$
Y_{\perp}(t)=z_{1}(0) e^{\lambda_{1} t}+z_{2}(0) e^{\lambda_{2} t}
$$

that depends on the initial conditions but not on the noise.

We will now apply the general theory to the model potential (60). Our aim is to expand the coordinates

$$
\begin{aligned}
& x(t)=X(t)+c \Delta x_{1}(t)+c^{2} \Delta x_{2}(t)+\cdots, \\
& y(t)=Y(t)+c \Delta y_{1}(t)+c^{2} \Delta y_{2}(t)+\cdots
\end{aligned}
$$

in powers of the anharmonicity parameter $c$. For expansions of other quantities, such as

$$
V^{\ddagger}=V_{0}^{\ddagger}+c V_{1}^{\ddagger}+c^{2} V_{2}^{\ddagger}+\cdots,
$$

a similar notation will be used. The anharmonic forces are given by

$$
\begin{aligned}
f_{x} & =-2 c x y^{2} \\
& =-2 c X Y^{2}-2 c^{2}\left(Y^{2} \Delta x_{1}+2 X Y \Delta y_{1}\right)+\cdots,
\end{aligned}
$$

$$
\begin{aligned}
f_{y} & =-2 c x^{2} y \\
& =-2 c X^{2} Y-2 c^{2}\left(2 X Y \Delta x_{1}+X^{2} \Delta y_{1}\right)+\cdots .
\end{aligned}
$$

In the first step of the iteration we find

$$
\begin{aligned}
\Delta u_{1}(t) & =\frac{1}{\lambda_{\mathrm{u}}-\lambda_{\mathrm{s}}} S\left[\lambda_{\mathrm{u}}, f_{x, 1} ; t\right] \\
& =-\frac{2}{\lambda_{\mathrm{u}}-\lambda_{\mathrm{s}}} S\left[\lambda_{\mathrm{u}}, X Y^{2} ; t\right],
\end{aligned}
$$

where $f_{x, n}$ is the coefficient of $f_{x}$ of order $c^{n}$. From Eq. (70) we get

$$
\begin{aligned}
V_{1}^{\ddagger} & =\left(\lambda_{\mathrm{u}}-\lambda_{\mathrm{s}}\right) \Delta u_{1}(0) \\
& =-2 S\left[\lambda_{\mathrm{u}}, X Y^{2} ; 0\right] .
\end{aligned}
$$

The remaining coordinates need only be calculated if the second-order correction for the critical velocity is desired. We then obtain

$$
\begin{aligned}
& \Delta s_{1}(t)=-\Delta u_{1}(0) e^{\lambda_{\mathrm{s}} t}+\frac{2}{\lambda_{\mathrm{u}}-\lambda_{\mathrm{s}}} \bar{S}\left[\lambda_{\mathrm{s}}, X Y^{2} ; t\right], \\
& \Delta z_{1}(t)=-\frac{2}{\lambda_{1}-\lambda_{2}} \bar{S}\left[\lambda_{1}, X^{2} Y ; t\right], \\
& \Delta z_{2}(t)=+\frac{2}{\lambda_{1}-\lambda_{2}} \bar{S}\left[\lambda_{2}, X^{2} Y ; t\right] .
\end{aligned}
$$

Finally, with the aid of

$$
\begin{aligned}
& \Delta x_{1}=\Delta u_{1}+\Delta s_{1}, \\
& \Delta y_{1}=\Delta z_{1}+\Delta z_{2},
\end{aligned}
$$

we can calculate

$$
\Delta u_{2}(t)=\frac{1}{\lambda_{\mathrm{u}}-\lambda_{\mathrm{s}}} S\left[\lambda_{\mathrm{u}}, f_{x, 2} ; t\right] .
$$

The resulting expression reduces to

$$
\begin{aligned}
V_{2}^{\ddagger}= & -4 S_{\tau}\left[\lambda_{\mathrm{u}}, \frac{Y^{2}(\tau)}{\lambda_{\mathrm{u}}-\lambda_{\mathrm{s}}}\left(S\left[\lambda_{\mathrm{u}}, X Y^{2} ; 0\right] e^{\lambda_{\mathrm{s}} \tau}-S\left[\lambda_{\mathrm{u}}, X Y^{2} ; \tau\right]\right.\right. \\
& \left.+\bar{S}\left[\lambda_{\mathrm{s}}, X Y^{2} ; \tau\right]\right)+2 \frac{X(\tau) Y(\tau)}{\lambda_{1}-\lambda_{2}}\left(\bar{S}\left[\lambda_{2}, X^{2} Y ; \tau\right]\right. \\
& \left.\left.-\bar{S}\left[\lambda_{1}, X^{2} Y ; \tau\right]\right) ; 0\right] .
\end{aligned}
$$

Figure 8 shows the value of the critical velocity for one realization of the noise for the two-dimensional model potential (60) as a function of the coupling strength, $c$, for the initial condition $y=0, v_{y}=0$. It is compared to perturbative results up to second order. As it can be seen, our perturbative results agree very well with those obtained numerically, thus showing the efficiency of our method. To further analyze the performance of our method, we show in Fig. 9 the difference between the numerically calculated critical velocity and the value obtained with our perturbative expansions for different values of the transverse coordinates, where it is clearly seen that it sensibly reduces as the order of the perturbation is increased. 


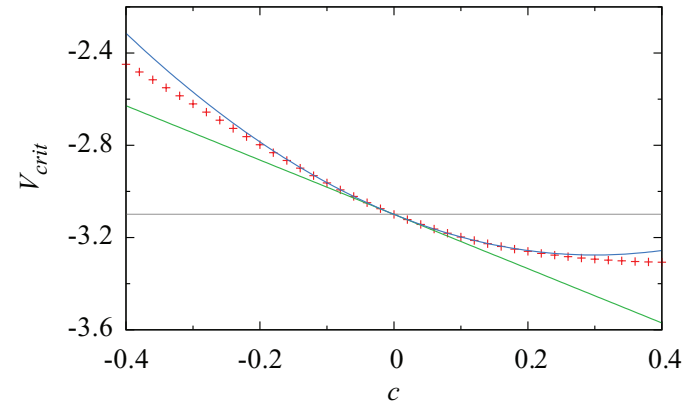

FIG. 8. Critical velocity for one realization of the noise for the twodimensional model potential (60) with $\omega_{x}=1, \omega_{y}=1.5, \gamma=2, k_{\mathrm{B}} T=1$, for an initial condition $y=0, v_{y}=0$. Numerical simulation results (red crosses), harmonic approximation (23) (gray horizontal line), perturbative results to first-order $(23)+(71)$ (green straight line), and second-order $(23)+(71)$ + (72) (blue line).

\section{Reaction rate expressions}

The simple expression (40) for the transmission coefficient in terms of the critical velocity can easily be generalized to higher dimension. To achieve this, we start again from Eq. (7). Note first that in the denominator of Eq. (7) the average over the transverse coordinates has no effect since the TST approximation to the characteristic function does not depend on them. In the numerator, we use again the form (39) of the characteristic function and carry out the average over $v_{x}$ as before, to obtain

$$
\kappa=\left\langle\exp \left(-\frac{V^{\ddagger 2}}{2 k_{\mathrm{B}} T}\right)\right\rangle_{\alpha \perp} .
$$

In this expression the average over the transverse coordinates, which is indicated by subscript $\perp$, cannot be carried out immediately because the critical velocity depends on the transverse coordinates.

Equation (73) represents the simplest conceivable generalization of Eq. (40). It is remarkable that no modifications, beyond the additional average over the transverse coordinates, are required. This is only possible because no anharmonic corrections are required for the denominator in Eq. (7).

In the case of the model potential (60), the distribution (4) of the transverse coordinates is given by

$$
p_{\perp}\left(y, v_{y}\right)=\frac{1}{Z} \exp \left(-\frac{v_{y}^{2}+\omega_{y}^{2} y^{2}}{2 k_{\mathrm{B}} T}\right),
$$

i.e., it is a Gaussian distribution. The functions $X$ and $Y$ will then both have a Gaussian distribution, which allows us to evaluate the rate corrections by the method of Sec. V B. For any expression involving $u^{\ddagger}(0), X$ and $Y$, we write

$$
\langle P(\ldots)\rangle_{\alpha \perp}=\frac{\lambda_{\mathrm{u}}}{\omega_{\mathrm{b}}}\langle\ldots\rangle_{0 \perp}
$$

as in Eq. (50). The average over the initial conditions is not involved in the transition from the noise average to the distorted average with correlation function (51), because the noise and the initial conditions are uncorrelated.

Once we have a perturbative expansion of the critical velocity of the form (41), expressions (43) can be used for the expansion of the transmission factor, the only required modifi-

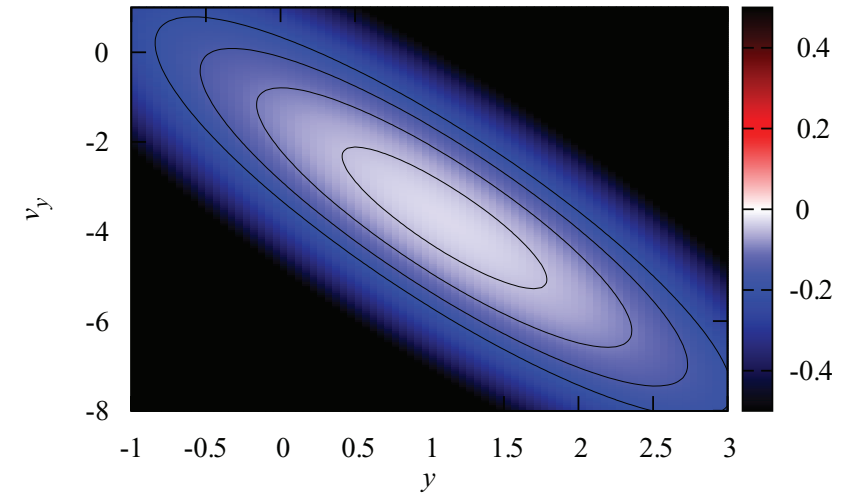

(a)

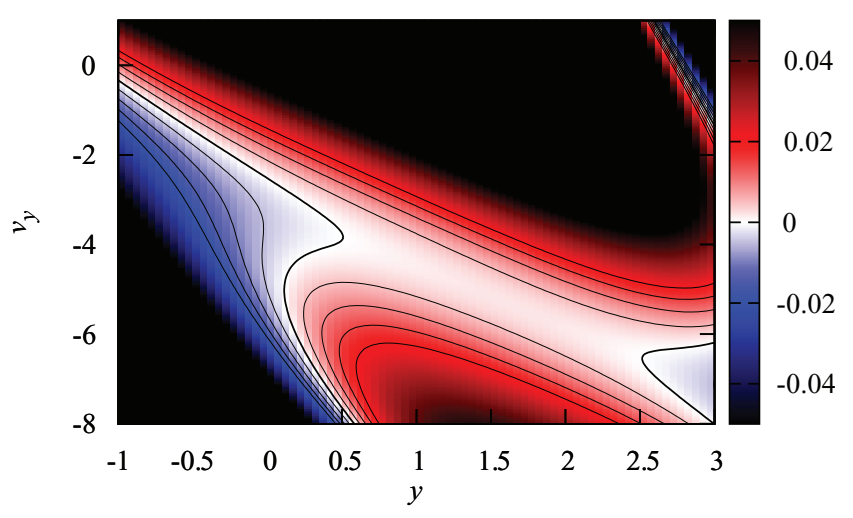

(b)

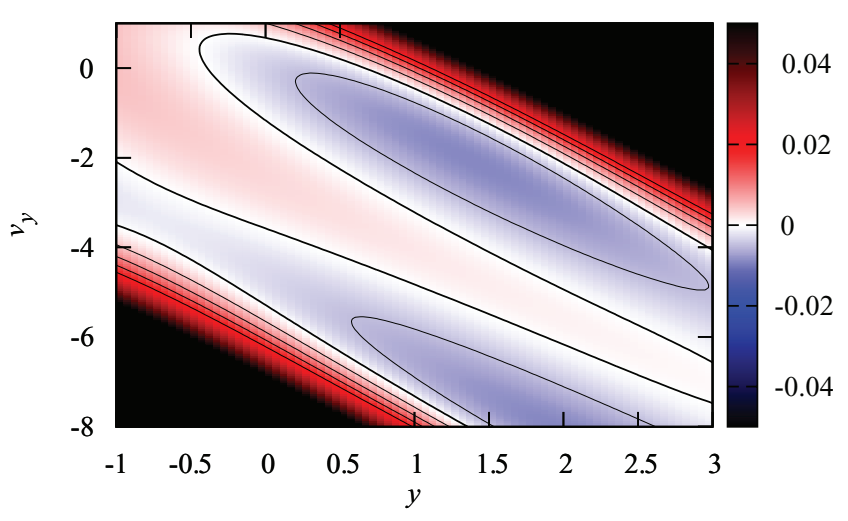

(c)

FIG. 9. Difference between numerically calculated critical velocity and perturbative expansions. Noise sequence and parameter values as in Fig. 7. (a) Harmonic approximation. (b) First-order perturbation theory. (c) Secondorder perturbation theory. Contour spacing is 0.05 in (a), 0.005 in (b) and (c). Note that the color scale is also stretched by a factor 10 in (a).

cation being to replace noise averages by averages over noise and the transverse coordinates.

Assuming a general anharmonic potential of the form

$$
U(0, y)=\frac{1}{2} \omega_{y}^{2} y^{2}+U_{\mathrm{anh}}(y),
$$

where $U_{\text {anh }}(y)$ contains terms at least of third order in $y$, i.e., at least of first order in the expansion parameter $\varepsilon$, it can be treated perturbatively in the current framework. The distribution function of the transverse coordinates can then be 
expanded as

$$
\begin{aligned}
p_{\perp}\left(y, v_{y}\right)= & \frac{1}{Z} \exp \left(-\frac{v_{y}^{2}+\omega_{y}^{2} y^{2}}{2 k_{\mathrm{B}} T}\right) \\
& \times\left(1+\varepsilon a_{1}(y)+\varepsilon^{2} a_{2}(y)+\cdots\right)
\end{aligned}
$$

with suitable coefficients $a_{i}$ that are polynomials in $y$ of degree at most $i$. We assume that the partition function $Z$ in Eq. (76) is the same as in the Gaussian distribution (74), and any corrections to the partition function that arise from the anharmonicity of the potential have been included in the expansion coefficients $a_{i}(y)$.

Using symbol $\Perp$ to denote an average over the Gaussian distribution (74) of initial conditions, we can write

$$
\begin{aligned}
\kappa & =\left\langle\exp \left(-\frac{V^{\ddagger 2}}{2 k_{\mathrm{B}} T}\right)\right\rangle_{\alpha \perp} \\
& =\left\langle\exp \left(-\frac{V^{\ddagger 2}}{2 k_{\mathrm{B}} T}\right) \times\left(1+\varepsilon a_{1}(y)+\varepsilon^{2} a_{2}(y)+\cdots\right)\right\rangle_{\alpha \Perp} .
\end{aligned}
$$

The expansion (41) of the critical velocity then allows us to expand the exponential, thus obtaining

$$
\kappa=\kappa_{0}+\varepsilon \kappa_{1}+\varepsilon^{2} \kappa_{2}+\cdots
$$

with

$$
\begin{gathered}
\kappa_{0}=\langle P\rangle_{\alpha \Perp}, \\
\kappa_{1}=-\frac{1}{k_{\mathrm{B}} T}\left\langle P V_{0}^{\ddagger} V_{1}^{\ddagger}\right\rangle_{\alpha \Perp}+\left\langle P a_{1}(y)\right\rangle_{\alpha \Perp}, \\
\kappa_{2}=\frac{1}{2\left(k_{\mathrm{B}} T\right)^{2}}\left\langle P V_{0}^{\ddagger 2} V_{1}^{\ddagger 2}\right\rangle_{\alpha \Perp}-\frac{1}{k_{\mathrm{B}} T}\left\langle P V_{0}^{\ddagger} V_{2}^{\ddagger}\right\rangle_{\alpha \Perp} \\
-\frac{1}{2 k_{\mathrm{B}} T}\left\langle P V_{1}^{\ddagger 2}\right\rangle_{\alpha \Perp}-\frac{1}{k_{\mathrm{B}} T}\left\langle P V_{0}^{\ddagger} V_{1}^{\ddagger} a_{1}(y)\right\rangle_{\alpha \Perp}+\left\langle P a_{2}(y)\right\rangle_{\alpha \Perp},
\end{gathered}
$$

where again the abbreviation (44) has been used. The remaining averages are Gaussian averages that can be evaluated, as before, by first converting the noise average into a distorted Gaussian average via (75), and then using Isserlis' theorem.

Because the factor $P$ is independent of the initial conditions, we obtain from (77a)

$$
\kappa_{0}=\langle P\rangle_{\alpha}=\frac{\lambda_{\mathrm{u}}}{\omega_{\mathrm{b}}}
$$

the Kramers result. Similarly, the expressions $\left\langle P a_{i}(y)\right\rangle_{\alpha \Perp}$, that occur in all correction terms, can be simplified to

$$
\left\langle P a_{i}(y)\right\rangle_{\alpha \Perp}=\langle P\rangle_{\alpha}\left\langle a_{i}(y)\right\rangle_{\Perp}=\frac{\lambda_{\mathrm{u}}}{\omega_{\mathrm{b}}}\left\langle a_{i}(y)\right\rangle_{\Perp} .
$$

\section{Correlation functions}

To evaluate corrections to the transmission factor in Eq. (77) using Isserlis' theorem, the correlation functions $\left\langle w_{1} w_{2}\right\rangle_{0 \Perp}$, where $w_{1}$ and $w_{2}$ are one of $u^{\ddagger}(0), X(t), Y(t)$, and $y(0)$, are needed. (The initial condition $y(0)$ was written without its time argument in Sec. VI C. For the sake of clarity we will now include it again.)

Because the $x$ and $y$ components of the fluctuating force are uncorrelated, all correlation functions involving one of either $u^{\ddagger}(0)$ or $X(t)$ and one of either $Y(t)$ or $y(0)$ must vanish. Furthermore, since $u^{\ddagger}(0)$ and $X(t)$ do not depend on initial conditions,

$$
\left\langle u^{\ddagger}(0) X(t)\right\rangle_{0 \Perp}=\left\langle u^{\ddagger}(0) X(t)\right\rangle_{0}
$$

and

$$
\left\langle X(t) X\left(t^{\prime}\right)\right\rangle_{0 \Perp}=\left\langle X(t) X\left(t^{\prime}\right)\right\rangle_{0}
$$

are given by Eq. (52).

Concerning the initial conditions, it can be read off from the distribution function (74) that

$$
\left\langle y(0)^{2}\right\rangle_{0 \Perp}=\frac{k_{\mathrm{B}} T}{\omega_{y}^{2}} .
$$

(The average over the distorted noise distribution does not have any effect.) We can also see that

$$
\left\langle v_{y}(0)^{2}\right\rangle_{0 \Perp}=k_{\mathrm{B}} T \quad \text { and } \quad\left\langle y(0) v_{y}(0)\right\rangle_{0 \Perp}=0 .
$$

These results further yield

$$
\begin{aligned}
\langle y(0) Y(t)\rangle_{0 \Perp} & =\left\langle y(0) Y_{\perp}(t)\right\rangle_{0 \Perp} \\
& =\left\langle y(0) z_{1}(0)\right\rangle_{0 \Perp} e^{\lambda_{1} t}+\left\langle y(0) z_{2}(0)\right\rangle_{0 \Perp} e^{\lambda_{2} t} \\
& =\frac{k_{\mathrm{B}} T}{\omega_{y}^{2}\left(\lambda_{1}-\lambda_{2}\right)}\left(\lambda_{1} e^{\lambda_{2} t}-\lambda_{2} e^{\lambda_{1} t}\right) .
\end{aligned}
$$

Finally, the autocorrelation function of $Y(t)$ can be decomposed, with the help of the split (69), into

$$
\left\langle Y(t) Y\left(t^{\prime}\right)\right\rangle_{0 \Perp}=\left\langle Y_{\alpha}(t) Y_{\alpha}\left(t^{\prime}\right)\right\rangle_{\alpha}+\left\langle Y_{\perp}(t) Y_{\perp}\left(t^{\prime}\right)\right\rangle_{\Perp}
$$

because

$$
\left\langle Y_{\alpha}(t) Y_{\perp}\left(t^{\prime}\right)\right\rangle_{0 \Perp}=\left\langle Y_{\alpha}(t)\right\rangle_{0}\left\langle Y_{\perp}\left(t^{\prime}\right)\right\rangle_{\perp}=0
$$

and

$$
\left\langle Y_{\alpha}(t) Y_{\alpha}\left(t^{\prime}\right)\right\rangle_{0}=\left\langle Y_{\alpha}(t) Y_{\alpha}\left(t^{\prime}\right)\right\rangle_{\alpha} .
$$

To evaluate the first term in Eq. (81), the correlation function of the components $z_{i}^{\ddagger}(t)$ of the TS trajectory, given in Ref. 31, are needed. The second term can be evaluated with the help of Eqs. (78) and (79). Finally, one arrives to the following simple result:

$$
\left\langle Y(t) Y\left(t^{\prime}\right)\right\rangle_{0 \Perp}=\frac{k_{\mathrm{B}} T}{\omega_{y}^{2}-\lambda_{1}^{2}} e^{\lambda_{1}\left|t-t^{\prime}\right|}+\frac{k_{\mathrm{B}} T}{\omega_{y}^{2}-\lambda_{2}^{2}} e^{\lambda_{2}\left|t-t^{\prime}\right|} .
$$

With that we have found all correlation functions that we will need to calculate the rate corrections.

\section{E. Rate corrections}

Let us now derive an expansion of the transmission factor for the case of the anharmonic model potential (60),

$$
\kappa=\kappa_{0}+c \kappa_{1}+c^{2} \kappa_{2}+\cdots,
$$




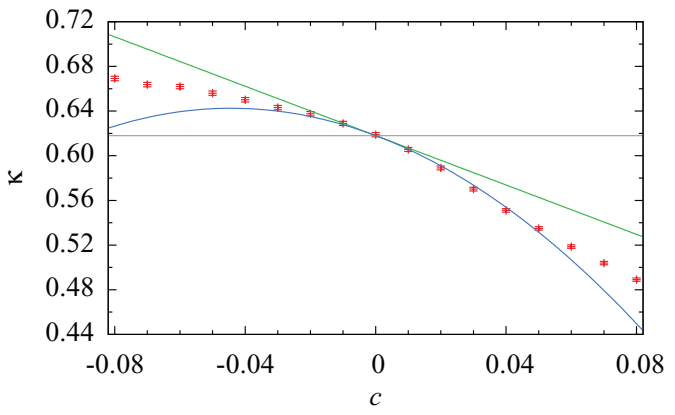

FIG. 10. Transmission factor for the two-dimensional model potential (60) as a function of coupling strength, $c$, for $\omega_{\mathrm{b}}=1, \omega_{y}=0.5, k_{\mathrm{B}} T=1$, $\gamma=1$. Numerical simulation results (red points), harmonic (Kramers) approximation (53) (gray horizontal line), perturbative results to first-order, obtained from $(53)+(85)$ (green line), and second-order obtained from (53) $+(85)+(86)$ (blue line).

in powers of the coupling parameter $c$. As discussed earlier, this corresponds to an expansion in powers of $\varepsilon^{2}$, and the rate formulas (77) with $a_{i}(y)=0$ can be used.

The first correction term is

$$
\begin{aligned}
\kappa_{1} & =-\frac{1}{k_{\mathrm{B}} T} \frac{\lambda_{\mathrm{u}}}{\omega_{\mathrm{b}}}\left\langle V_{0}^{\ddagger} V_{1}^{\ddagger}\right\rangle_{0 \Perp} \\
& =-\frac{2}{k_{\mathrm{B}} T} \frac{\lambda_{\mathrm{u}}}{\omega_{\mathrm{b}}}\left(\lambda_{\mathrm{u}}-\lambda_{\mathrm{s}}\right) S_{\tau}\left[\lambda_{\mathrm{u}},\left\langle u^{\ddagger}(0) X(\tau) Y^{2}(\tau)\right\rangle_{0 \Perp} ; 0\right] .
\end{aligned}
$$

The remaining average can be simplified to

$$
\left\langle u^{\ddagger}(0) X(\tau) Y^{2}(\tau)\right\rangle_{0 \Perp}=\left\langle u^{\ddagger}(0) X(\tau)\right\rangle_{0}\left\langle Y^{2}(\tau)\right\rangle_{0 \Perp} .
$$

The results of Sec. VI D give a sum of exponentially decaying terms for this expression, so that the $S$ functional can be evaluated as in the one-dimensional case. In terms of the dimensionless parameters $\mu=\kappa_{0}=\lambda_{\mathrm{u}} / \omega_{\mathrm{b}}$, that was already used above, and $v=\omega_{y} / \omega_{\mathrm{b}}$ the rate correction reads

$$
\kappa_{1}=-\frac{\gamma k_{\mathrm{B}} T}{\omega_{\mathrm{b}}^{5}} \frac{\mu^{2}}{(1+\mu)^{2} v^{2}} .
$$

The second-order correction can be obtained in a similar way. After tedious calculations, one finally arrives at

$$
\begin{aligned}
\kappa_{2}= & \frac{\mu\left(k_{\mathrm{B}} T\right)^{2}}{6 \omega_{\mathrm{b}}^{8}}\left(\frac{96\left(\mu^{2}-1\right)^{2}}{\left(\mu^{2}+1\right)^{2}\left(\mu^{2}-4 v^{2}-2\right)}-\frac{6}{\left(\mu^{2}+1\right)\left(v^{2}+1\right)}\right. \\
& -\frac{16}{\left(2 \mu^{2}+1\right)\left(3 \mu^{2}+4 v^{2}+6\right)}+\frac{9\left(\mu^{2}-1\right)\left(3 \mu^{4}+8 \mu^{2}+1\right)}{\left(\mu^{2}+1\right)^{3} v^{4}} \\
& +\frac{64 \mu^{8}}{\left(\mu^{2}+1\right)^{2}\left(\mu^{2}+2\right)\left(\mu^{4}-2 \mu^{2}\left(2 v^{2}+1\right)-8\right)} \\
& -\frac{96\left(\mu^{4}+2 \mu^{2}-1\right) \mu^{6}}{\left(\mu^{2}+1\right)^{4}\left(\mu^{4}-2 \mu^{2}\left(2 v^{2}+1\right)-3\right)} \\
& +\frac{192 \mu^{6}}{\left(2 \mu^{6}+7 \mu^{4}+7 \mu^{2}+2\right)\left(\mu^{2}\left(4 v^{2}+6\right)+3\right)} \\
& \left.+\frac{2\left(16 \mu^{12}-24 \mu^{10}-139 \mu^{8}-75 \mu^{6}+77 \mu^{4}+111 \mu^{2}+34\right)}{\left(\mu^{2}+1\right)^{4}\left(2 \mu^{4}+5 \mu^{2}+2\right) v^{2}}\right) .
\end{aligned}
$$

A numerical example is shown in Fig. 10. The secondorder corrections to the transmission coefficient are small, so that a large number of trajectories needs to be included in the numerical calculation of the rate. Nevertheless, it is clear that the perturbative expressions (85) and (86) describe the rate correctly.

\section{CONCLUDING REMARKS}

TST and related schemes have been widely used for rate calculations for a long time. For reactions that occur in solution, recrossings of the DS pose a major difficulty in such calculations. Many approaches try to overcome this problem by choosing the DS judiciously. By contrast, the method developed here is insensitive to the choice of this surface. The simplest choice of DS, which was taken here, also leads to the simplest calculation of the critical velocity. The use of a different DS would require a redefinition of the critical velocity to describe its intersection with the stable manifold, but this can be achieved with only minor modifications to the iteration procedure for the critical velocity. After that, any DS that lies within the barrier region would give the same rate.

This independence of the DS is achieved by two crucial features of our method. First, the dynamics are described in phase space, rather than in configuration space, and modern geometric methods are used in our study. Second, we focus on invariant geometric structures that are determined by the dynamics, rather than in structures, such as the DS, that are arbitrarily imposed by the researcher. The present results indicate that similar results apply to reactive systems that are coupled to their environments, i.e., TST should focus on invariant structures in phase space.

The focus of the present paper has been on analytic perturbation theory for the rate corrections on an anharmonic barrier. The different steps of this calculation have different levels of complexity. The critical velocity, which encodes the location of the invariant manifold, is very simple to calculate with the iteration scheme described here. Moreover, it can easily be extended to higher orders. By contrast, the evaluation of the averages that yield the rate corrections is laborious. While straightforward in principle, it requires the calculation of a large number of exponential integrals, something that, even for some of the results presented here, is only feasible with the help of a computer algebra system, Mathematica ${ }^{45}$ in our case.

The crucial step that sets the current method apart from earlier algorithms is the calculation of the stable manifold and the critical velocity. It is encouraging, therefore, that this most important step of the calculation is also the easiest. This observation further suggests that to obtain an efficient algorithm to compute rates, the calculation of the stable manifold should be combined with numerical methods for the computation of averages. We will report on such combinations in a forthcoming publication.

\section{ACKNOWLEDGMENTS}

This work has been supported by the MCINN (Spain) under projects MTM2009-14621 and CONSOLIDER 2006-32 
(i-Math). F.R. gratefully acknowledges a doctoral fellowship the UPM and the hospitality of the members of the School of Mathematics at Loughborugh University, where part of this work was done.

${ }^{1}$ D. G. Truhlar, W. L. Hase, and J. T. Hynes, J. Phys. Chem. 87, 2664 (1983).

${ }^{2}$ D. G. Truhlar, B. C. Garrett, and S. J. Klippenstein, J. Phys. Chem. 100, 12771 (1996).

${ }^{3}$ W. H. Miller, Faraday Discuss. Chem. Soc. 110, 1 (1998).

${ }^{4}$ M. Toller, G. Jacucci, G. DeLorenzi, and C. P. Flynn, Phys. Rev. B 32, 2082 (1985).

${ }^{5}$ B. Eckhardt, J. Phys. A 28, 3469 (1995).

${ }^{6}$ R. Hernandez and W. H. Miller, Chem. Phys. Lett. 214, 129 (1993).

${ }^{7}$ R. Hernandez, J. Chem. Phys. 101, 9534 (1994).

${ }^{8}$ C. Jaffé, D. Farrelly, and T. Uzer, Phys. Rev. A 60, 3833 (1999).

${ }^{9}$ C. Jaffé, D. Farrelly, and T. Uzer, Phys. Rev. Lett. 84, 610 (2000).

${ }^{10}$ W. S. Koon, M. W. Lo, J. E. Marsden, and S. D. Ross, Chaos 10, 427 (2000).

${ }^{11}$ C. Jaffé, S. D. Ross, M. W. Lo, J. Marsden, D. Farrelly, and T. Uzer, Phys. Rev. Lett. 89, 011101 (2002).

${ }^{12}$ T. Uzer, C. Jaffé, J. Palacián, P. Yanguas, and S. Wiggins, Nonlinearity 15, 957 (2002).

${ }^{13}$ T. Komatsuzaki and R. S. Berry, Adv. Chem. Phys. 123, 79 (2002).

${ }^{14} \mathrm{H}$. Waalkens, A. Burbanks, and S. Wiggins, J. Phys. A 37, L257 (2004).

${ }^{15}$ H. Waalkens, A. Burbanks, and S. Wiggins, J. Chem. Phys. 121, 6207 (2004).

${ }^{16}$ B. C. Garrett, and D. G. Truhlar, in Theory and Applications of Computational Chemistry: The First Forty Years, edited by C. E. Dykstra, G. Frenking, K. S. Kim, and G. E. Scuseria (Elsevier, 2005), Chap. 5, pp. 67-87.

${ }^{17}$ P. Hänggi, P. Talkner, and M. Borkovec, Rev. Mod. Phys. 62, 251 (1990).

${ }^{18}$ P. Pechukas, in Dynamics of Molecular Collisions, Part B, edited by W. H. Miller (Plenum, New York, 1976), pp. 269-322.

${ }^{19}$ D. Chandler, J. Chem. Phys. 68, 2959 (1978).
${ }^{20}$ J. P. Bothma, J. B. Gilmore, and R. H. McKenzie, New J. Phys. 12, 055002 (2010).

${ }^{21}$ D. Polli, P. Altoè, O. Weingart, K. M. Spillane, C. Manzoni, D. Brida, G. Tomasello, G. Orlandi, P. Kukura, R. A. Mathies, M. Garavelli, and G. Cerullo, Nature (London) 467, 440 (2010).

${ }^{22}$ H. A. Kramers, Physica (Utrecht) 7, 284 (1940).

${ }^{23}$ R. Zwanzig, J. Stat. Phys. 9, 215 (1973).

${ }^{24}$ V. I. Mel'nikov and S. V. Meshkov, J. Chem. Phys. 85, 1018 (1986).

${ }^{25}$ E. Pollak, H. Grabert, and P. Hänggi, J. Chem. Phys. 91, 4073 (1989).

${ }^{26}$ E. Pollak and P. Talkner, Phys. Rev. E 47, 922 (1993).

${ }^{27}$ P. Talkner and E. Pollak, Phys. Rev. E 47, R21 (1993).

${ }^{28}$ P. Talkner, Chem. Phys. 180, 199 (1994).

${ }^{29}$ P. L. García-Müller, F. Borondo, R. Hernandez, and R. M. Benito, Phys. Rev. Lett. 101, 178302 (2008).

${ }^{30}$ T. Bartsch, R. Hernandez, and T. Uzer, Phys. Rev. Lett. 95, 058301 (2005).

${ }^{31}$ T. Bartsch, T. Uzer, and R. Hernandez, J. Chem. Phys. 123, 204102 (2005).

${ }^{32}$ T. Bartsch, T. Uzer, J. M. Moix, and R. Hernandez, J. Chem. Phys. 124, 244310 (2006).

${ }^{33}$ T. Bartsch, T. Uzer, J. M. Moix, and R. Hernandez, J. Phys. Chem. B 112, 206 (2008).

${ }^{34}$ R. Hernandez, T. Uzer, and T. Bartsch, Chem. Phys. 370, 270 (2010).

${ }^{35}$ F. Revuelta, T. Bartsch, R. M. Benito, and F. Borondo, J. Chem. Phys. 136, 091102 (2012)

${ }^{36}$ S. Kawai and T. Komatsuzaki, J. Chem. Phys. 131, 224505 (2009).

${ }^{37}$ S. Kawai and T. Komatsuzaki, J. Chem. Phys. 131, 224506 (2009).

${ }^{38}$ S. Kawai and T. Komatsuzaki, Phys. Chem. Chem. Phys. 12, 7626 (2010).

${ }^{39}$ S. Kawai and T. Komatsuzaki, Phys. Chem. Chem. Phys. 12, 7636 (2010).

${ }^{40}$ R. Zwanzig, Nonequilibrium Statistical Mechanics (Oxford University Press, London, 2001).

${ }^{41}$ S. Kawai, A. D. Bandrauk, C. Jaffé, T. Bartsch, J. Palacián, and T. Uzer, J. Chem. Phys. 126, 164306 (2007).

${ }^{42}$ L. Arnold, Random Dynamical Systems (Springer, Berlin, 1998).

${ }^{43}$ L. Isserlis, Biometrika 11, 185 (1916).

${ }^{44}$ L. Isserlis, Biometrika 12, 134 (1918).

${ }^{45}$ S. Wolfram, The Mathematica Book (Wolfram Media, 2005). 